\title{
Convoluted Intake Distortion Measurements Using Stereo Particle Image Velocimetry
}

\author{
Daniel Gil-Prieto ${ }^{1}$, David G. MacManus ${ }^{2}$ (MAIAA), Pavlos K. Zachos ${ }^{3}$, and Geoffrey Tanguy ${ }^{4}$ \\ Cranfield University, Cranfield, England, MK43 OAL, United Kingdom \\ Kevin R. Menzies ${ }^{5}$ \\ Rolls-Royce plc, P O Box 3, Filton, Bristol, BS34 7QE, United Kingdom
}

The unsteady distorted flow fields generated within convoluted aero-engine intakes can compromise the engine performance and operability. Therefore there is a need for a better understanding of the complex characteristics of the distorted flow at the exit of S-shaped intakes. This work presents a detailed analysis of the unsteady swirl distortion based on synchronous, high spatial resolution measurements using stereoscopic particle image velocimetry. Two S-duct configurations with different centerline offsets are investigated. The high offset duct shows greater levels of dynamic and steady swirl distortion and a notably greater tendency towards bulk swirl patterns associated with high swirl distortion. More discrete distortion patterns with locally high swirl levels and the potential to impact the engine operability are identified. The most energetic coherent structures of the flow field are observed using proper orthogonal decomposition. A switching mode is identified which promotes the alternating swirl switching mechanism and is mostly associated with the occurrence of potent bulk swirl events. A vertical mode which characterizes a perturbation of the vertical velocity field promotes most of the twin swirl flow distortion topologies. It is postulated that it is associated with the unsteadiness of the centerline shear layer.

\section{Nomenclature}

$a$ $=$ POD temporal coefficient, $\mathrm{m} / \mathrm{s}$

${ }^{1}$ PhD Student, Propulsion Engineering Centre, Building 52, Cranfield University.

${ }^{2}$ Senior Lecturer, Propulsion Engineering Centre, Building 52, Cranfield University, AIAA Member.

${ }^{3}$ Lecturer, Propulsion Engineering Centre, Building 52, Cranfield University, AIAA Member.

${ }^{4} \mathrm{PhD}$ Student, Propulsion Engineering Centre, Building 52, Cranfield University.

${ }^{5}$ Corporate Specialist - CFD, System Design 


$$
\begin{aligned}
& A R \quad=\text { Area Ratio } \\
& D \quad=\text { S-duct cross section diameter, } \mathrm{mm} \\
& h \quad=\text { S-duct centerline offset, } \mathrm{mm} \\
& H=\text { Boundary layer shape factor, } \delta^{*} / \theta \\
& L \quad=\quad \text { S-duct axial length, } \mathrm{mm} \\
& K E \quad=\quad \text { Kinetic Energy, } \mathrm{J} / \mathrm{kg} \\
& M \quad=\text { Mach number } \\
& P \quad=\text { Probability, } \% \\
& P D F^{*}=\text { Non-dimensional Probability Density Function } \\
& r \quad=\text { Radial coordinate from the AIP center, } \mathrm{mm} \\
& R=\text { S-duct cross section radius, } \mathrm{mm} \\
& R_{c}=\text { Curvature radius of the S-duct bend, } \mathrm{mm} \\
& R e_{D}=\text { Reynolds number based on the inlet diameter } \\
& s \quad=\quad \text { S-duct centerline co-ordinate, } \mathrm{mm} \\
& S D=\text { Swirl Directivity distortion descriptor } \\
& \text { SI }=\text { Swirl Intensity distortion descriptor, deg } \\
& S P=\text { Swirl Pairs distortion descriptor } \\
& \text { St } \quad=\text { Strouhal number, } f D_{A I P} /\left\langle\bar{w}_{A I P}\right\rangle \\
& S S_{i, k}^{+} \quad=\text { Positive Sector Swirl in the k-th swirling region of the } \mathrm{i} \text {-th ring } \\
& S S_{i, k}^{-}=\text {Negative Sector Swirl in the } \mathrm{k} \text {-th swirling region of the } \mathrm{i} \text {-th ring } \\
& \text { TKE = Turbulent Kinetic Energy, } \mathrm{J} / \mathrm{kg} \\
& u, v, w=\text { Velocity vector cartesian components, } \mathrm{m} / \mathrm{s} \\
& v_{\theta} \quad=\text { Circumferential velocity component, } \mathrm{m} / \mathrm{s} \\
& \alpha=\text { Swirl angle, } \arctan \left(v_{\theta} / w\right), \operatorname{deg} \\
& \alpha_{1} \quad=\text { Maximum sector swirl descriptor, deg } \\
& \delta \quad=\text { Thickness of the boundary layer where } w<0.99 w_{c l}, \mathrm{~mm} \\
& \delta^{*}=\text { Displacement thickness of the boundary layer, } \mathrm{mm} \\
& \gamma=\text { Curvature ratio based on the inlet-section radius, } R_{\text {in }} / R_{c} \\
& 2
\end{aligned}
$$$$
\text { American Institute of Aeronautics and Astronautics }
$$ 


$$
\begin{array}{ll}
\theta & =\text { Momentum thickness of the boundary layer, } \mathrm{mm} \\
\theta_{1} & =\text { Extent of the maximum sector swirl region, deg } \\
\Phi & =\text { POD non-dimensional modal distribution }
\end{array}
$$

\section{Subscripts}

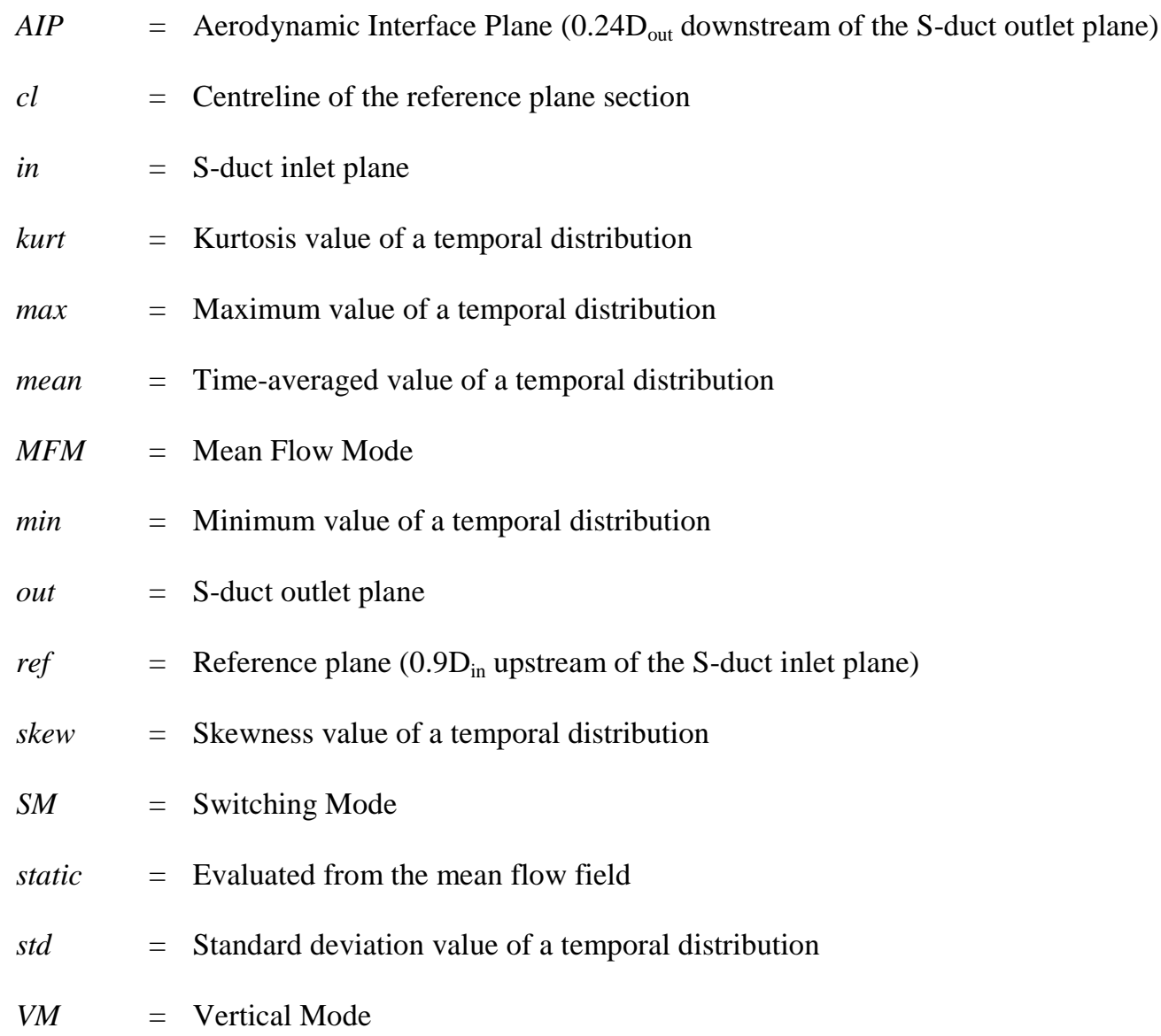

\section{Superscripts}

$V_{i p} \quad=$ In-plane velocity modulus, $\sqrt{u^{2}+v^{2}}$

$w \quad=\quad$ Stream-wise velocity field

\section{Operators}

$\begin{array}{ll}\langle\cdot\rangle & =\text { Time-average } \\ - & =\text { Area-average } \\ \sigma . & =\text { Standard deviation }\end{array}$


|. $\quad=$ Absolute value

\section{Introduction}

CONVOLUTED aero-engine intakes are expected to play a major role in the next generation of aircraft with embedded propulsion systems. Integrated engines may permit more efficient aircraft designs in terms of drag and weight, and are of interest to novel civil applications configurations ${ }^{1,2}$. However the intake configurations for these embedded engines can promote high levels of unsteady total pressure and swirl distortion which can adversely affect the engine performance, operability and structural integrity ${ }^{3}$. Unsteady total pressure and swirl distortion can be generated by flow separations and secondary flows within the intake. The negative effect of total pressure distortion on the turbomachinery components of the aero-engine has received notable attention ${ }^{4-8}$. The impact of swirl distortion has received relatively less attention since historically swirl issues were implicitly mitigated with inlet guide vanes and simple intake designs ${ }^{3}$. However previous work showed that for a system with inlet total-pressure distortion, the introduction of counter-rotating swirl substantially reduced the stability margin ${ }^{3}$. Previous studies noted that engine stall can be generated from distortion fluctuations ${ }^{9}$, and highlighted the importance of the turbulence levels and local peak distortion values ${ }^{6,10}$.

A number of previous studies have assessed the flow distortion associated with S-duct configurations. Wellborn et al. ${ }^{11}$ undertook an experimental investigation of the flow through a diffusing $\mathrm{S}$-duct $\left(\mathrm{AR}=1.52, \mathrm{~L} / \mathrm{D}_{\mathrm{in}}=5.0\right.$, $\mathrm{h} / \mathrm{L}=0.27$, see Fig. 1a) based on low-bandwidth instrumentation. A total pressure deficit was observed in the lower sector of the Aerodynamic Interface Plane (AIP) which was associated with the flow separation at the inner section of the first bend of the duct. Based on the time-averaged measurements, the presence of a pair of counter-rotating vortices promoted by the curvature of the duct was also reported. The flow separation and reattachment points were observed at centreline coordinates $\mathrm{s} / \mathrm{D}_{\mathrm{in}}=2.02$ and 4.13 , respectively. Fiola et al. ${ }^{12}$ assessed the performance of different turbulence models for the RANS simulation of the flow in the same S-duct studied by Wellborn et al. ${ }^{11}$. The four equation transition SST model provided the best match with the experimental data. However due to the high computational cost associated with this model, Fiola et al. ${ }^{12}$ chose the $\mathrm{k}-\omega$ SST model for further investigations since it provided similar results at a reasonable computational cost. Berens et al. ${ }^{13}$ performed a Detached Eddy Simulation (DES) of the flow in an S-duct with a similar offset ratio $\mathrm{h} / \mathrm{L}$ of $0.28\left(\mathrm{AR}=1.4, \mathrm{~L} / \mathrm{D}_{\mathrm{in}}=3.76\right)$. Time- 
averaged data was found to be misleading for the analysis of the highly unsteady S-duct flow. It was concluded that the unsteady nature of the flow distortion should be considered for a more reliable assessment of the engine/intake compatibility. Garnier ${ }^{14}$ investigated the performance of active flow control in an S-duct $\left(A R=1.52, L / \mathrm{D}_{\text {in }}=4.95\right.$, $\mathrm{h} / \mathrm{L}=0.50$ ) based on unsteady total pressure data acquired with 40 high-bandwidth transducers at the AIP. For the baseline uncontrolled flow at $\mathrm{M}_{\mathrm{AIP}}=0.2$, the greatest levels of total pressure fluctuations at the AIP were associated with a lateral oscillation of the low total pressure region, which occurred at a frequency of $\mathrm{St}=0.48$. Garnier ${ }^{14}$ also observed separated flow at the first inner bend of the S-duct. For the uncontrolled case at $\mathrm{M}_{\mathrm{AIP}}=0.20$, the separation point was identified at a centreline co-ordinate of $\mathrm{s} / \mathrm{D}_{\mathrm{in}}=2.17$, and the separation-bubble length of approximately $1.35 \mathrm{D}_{\text {in }}$ was estimated based on the static pressure distribution along the walls. The unsteady reattachment point fluctuations were associated with frequencies between $\mathrm{St}=0.48$ and 1.20. Zachos et al. ${ }^{15}$ used Stereoscopic Particle Image Velocimetry (S-PIV) to measure the distorted velocity field at the outlet of the two S-duct with the same nondimensional geometrical parameters (h/L, AR, L/D $\mathrm{D}_{\text {in }}$ ) as the configurations investigated by Garnier ${ }^{14}$ and Wellborn ${ }^{11}$, respectively. The main difference between these two configurations was the centreline offset which was $\mathrm{h} / \mathrm{L}=0.50$ and $h / L=0.27$, respectively. The inlet Mach number ranged from 0.27 to 0.60 , with a concomitant variation of $\operatorname{Re}_{D}$ between $5.9 \times 10^{5}$ and $13.8 \times 10^{5}$. Based on the high-spatial resolution, synchronous S-PIV measurements, Zachos et al. ${ }^{15}$ observed that the maximum fluctuations of the axial velocity occurred in the upper bounds of the mean-flow low axial velocity region, and were postulated to be associated with the shear layer unsteadiness. The swirl angle maximum fluctuations were found in the lower sector of the AIP, where the mean-flow was relatively swirl-free. Compared with the low-offset configuration, the high offset duct had notably higher levels of swirl distortion with 1.8 and 1.5 times greater values of mean and standard-deviation Swirl Intensity (SI), respectively. The SI distribution was positively skewed which indicated a greater likelihood of occurrence of adverse swirl distortion events. Notable excursions from the mean-flow twin swirl pattern were observed towards single swirling flow patterns rotating in either clockwise or anti-clockwise direction. The inlet Mach number had a modest effect on the overall characteristics of both steady and fluctuating flow fields, as well as in the swirl descriptors statistics, for the range investigated between 0.27 and 0.60 . For example, $\mathrm{SI}_{\text {std }}$ increased from $1.6^{\circ}$ to $1.7^{\circ}$, and $\mathrm{SI}_{\max }$ increased from $14.9^{\circ}$ to $15.6^{\circ}$ across this range of inlet Mach number for the high offset duct $(\mathrm{h} / \mathrm{L}=0.50) . "$

MacManus et al. ${ }^{16}$ performed a Delayed Detached Eddy Simulation (DDES) of the flow field in two S-ducts which corresponded to the geometry investigated by Garnier ${ }^{14}$ and a scaled version of the geometry investigated by 5 American Institute of Aeronautics and Astronautics 
Wellborn et al. ${ }^{11}$. The main difference between these S-duct configurations was the centreline offset which was $\mathrm{h} / \mathrm{L}=0.50$ and $\mathrm{h} / \mathrm{L}=0.27$, respectively. Two Mach numbers were simulated for each configuration, $\mathrm{M}_{\mathrm{AIP}}=0.18$ and $\mathrm{M}_{\mathrm{AIP}}=0.36$, which resulted in $\mathrm{Re}_{\mathrm{D}}$ of approximately $1.1 \times 10^{6}$ and $1.7 \times 10^{6}$, respectively. MacManus et al. ${ }^{16}$ calculated the Proper Orthogonal Decomposition (POD) of the total-pressure field to identify the dominant flow coherent structures. For these two configurations the two dominant flow structures were a lateral and a vertical oscillation of the low total pressure region observed in the time-averaged total pressure field. It was proposed that the lateral oscillation was associated with the secondary flows, while the vertical perturbation was related to the unsteadiness of the centreline diffusion-driven separation. The Mach number did not alter the characteristics of the most energetic structures of the flow field. For the low offset duct $(\mathrm{h} / \mathrm{L}=0.27)$, the spectral analysis revealed the lateral and vertical oscillations occurred at about $\mathrm{St}=0.4$ and $\mathrm{St}=0.75$, respectively, for both $\mathrm{M}_{\mathrm{AIP}}=0.18$ and $\mathrm{M}_{\mathrm{AIP}}=0.36$. For the high offset duct $(\mathrm{h} / \mathrm{L}=0.50)$ at $\mathrm{M}_{\mathrm{AIP}}=0.18$, the dominant frequencies for the lateral and vertical oscillations were $\mathrm{St}=0.55$ and $\mathrm{St}=0.9$, respectively. At $\mathrm{M}_{\mathrm{AIP}}=0.36$ the $S t$ associated with the lateral perturbation was approximately $\mathrm{St}=0.55$ as well, while the unsteadiness associated with the stream-wise separation showed a more broadband spectral content ${ }^{16}$.

The flow in an S-duct shows similarities with the flow in curved pipes, even though in the latter investigations the cross-section is usually constant and the flow typically undertakes a single $90^{\circ}$ bend. These flows are of interest for a wide range of engineering applications, such as gas and oil pipelines, heat exchangers and internal combustion engines ${ }^{17}$. As in S-ducts, the mean flow downstream of a $90^{\circ}$ bend is characterised by the presence of a symmetric pair of counter rotating vortices, referred to as Dean vortices ${ }^{17}$. In turbulent flows the Dean vortices oscillate so that one of them alternately dominates the flow field and this oscillatory mechanism was referred to as swirl switching ${ }^{18}$. Rutten et al. ${ }^{19}$ performed Large Eddy Simulations of the air-flow in two $90^{\circ}$ bends with curvature ratios $\left(R / R_{c}\right)$ of $\gamma=0.17$ and $\gamma=0.5$, respectively. The Reynolds number ranged between $\operatorname{Re}_{\mathrm{D}}=5 \times 10^{3}$ and $2.7 \times 10^{4}$. As opposed to the rapid switching between two stable states postulated by Tunstall and Harvey ${ }^{18}$, Rutten et al. ${ }^{19}$ observed that the swirl switching occurred as a result of a smooth transition between states. Kalpakli Vester et al. ${ }^{17}$ used Time-Resolved SPIV (TR-S-PIV) to measure the air-flow velocity field downstream of a $90^{\circ}$ bend for two geometries with curvature ratios of $\gamma=0.14$ and $\gamma=0.39$, at a $\operatorname{Re}_{\mathrm{D}}=2.3 \times 10^{4}$. POD was applied on the in-plane velocity field and the so-called switching mode showed a single rotating cell which promoted the swirl switching oscillation of the Dean vortices. This switching mode had a dominant frequency at $\mathrm{St}=0.1$ and a smaller peak at $\mathrm{St}=0.04$. The switching mode was also observed by Hellström et al. ${ }^{20}$ who used TR-S-PIV for the investigation of the water flow in a $90^{\circ}$ bend with a 
curvature ratio of $\gamma=0.5$ at $\operatorname{Re}_{\mathrm{D}}=2.5 \times 10^{4}$. The frequency associated with the switching mode was in this case of $\mathrm{St}=0.33$.

The need to consider the instantaneous distorted flow field highlights the limitations of conventional intake/engine compatibility assessments based on time-averaged data. S-PIV permits the measurement of synchronous velocity fields which enables he assessment of the unsteady distorted flow field. An additional benefit of S-PIV is the much greater spatial resolution, about 200 times $^{15}$, compared with conventional measuring techniques which are typically based on 40 pressure probes. This is of paramount importance to characterise the complex unsteady distorted patterns observed at the outlet of S-ducts, which can result in a loss of surge margin and engine operability concerns. The aim of the present investigation is to use S-PIV measurements to characterise the swirl distortion pattern generated within S-ducts.

The present study follows on from the initial work by Zachos et al. ${ }^{15}$ and MacManus et al. ${ }^{16}$, and provides a more detailed analysis of the effect of the duct offset $(\mathrm{h} / \mathrm{L})$ on the dynamic distortion characteristics. The POD analysis by MacManus et al. ${ }^{16}$ was applied to the computed total pressure field in similar configurations. In the present investigation POD is applied to the three components of the measured velocity vector. This enables the identification of the in-plane and out-of-plane characteristics of the main coherent structures in S-duct intakes, and helps to develop the understanding of the dominant flow structures in these flow fields. The effect of these coherent structures in the swirl distortion characteristics is quantified for the first time, both in terms of swirl pattern and intensity. In terms of distortion assessment, the cloud maps relating different distortion descriptors presented in previous investigations ${ }^{14,15}$ are further developed as joint Probability Density Function (PDF) maps, which permit the assessment of the likelihood of the distortion pattern associated with each region of the maps. A novel distortion analysis based on the characterisation of the most spoiled swirling region permits the identification of potent local distortion events, for which only a reduced region of the AIP is affected by the presence of distorted flow. These local events which are not highlighted by the conventional SI, SD, SP swirl distortion descriptors ${ }^{3}$ are important since they can drive engine instabilities when the associated extent exceeds a critical threshold ${ }^{8}$. Finally, the effect of the radial position on the unsteady distortion characteristics at the AIP is also assessed. 


\section{Methodology}

\section{A. Test cases}

The S-duct geometries and flow conditions are the same as those reported by Zachos et al. ${ }^{15}$. Duct-1 (Fig. 1) is a scaled-down version of the geometry investigated by Garnier ${ }^{14}$. Duct-2 non-dimensional geometry is similar to the configuration investigated by Wellborn et al. ${ }^{11}$. Both S-ducts present a circular cross-section and the main difference is the offset which is $\mathrm{h} / \mathrm{L}=0.50$ and $\mathrm{h} / \mathrm{L}=0.27$ for Duct- 1 and Duct- 2 respectively (Fig. $1 b$ ). The high offset Duct- 1 $(\mathrm{h} / \mathrm{L}=0.50)$ is composed of two consecutive $52^{\circ}$ bends with curvature ratios of $\gamma=0.16$, based on the inlet radius. The low offset Duct-2 $(\mathrm{h} / \mathrm{L}=0.27)$ consists of two $30^{\circ}$ bends of $\gamma=0.10$.

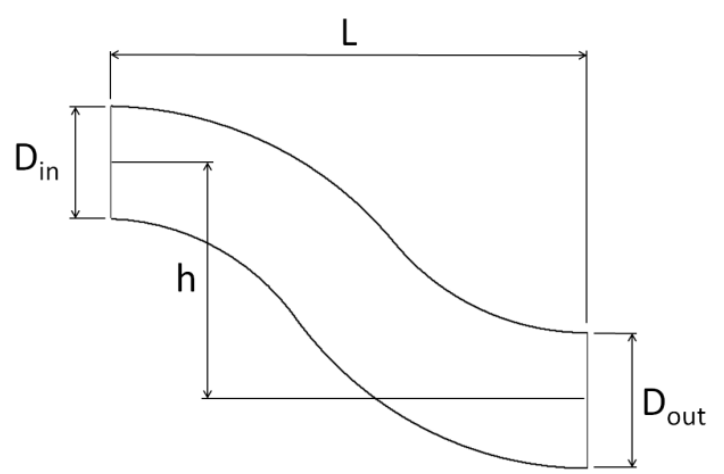

a) Schematic definition of S-duct geometrical parameters

\begin{tabular}{ccc}
\hline \hline Parameter & $\begin{array}{c}\text { High offset } \\
\text { (Duct-1) }\end{array}$ & $\begin{array}{c}\text { Low offset } \\
\text { (Duct-2) }\end{array}$ \\
\hline $\mathrm{D}_{\text {in }}$ & $121.6 \mathrm{~mm}$ & $121.6 \mathrm{~mm}$ \\
$\mathrm{~A}_{\text {out }} / \mathrm{A}_{\text {in }}$ & 1.52 & 1.52 \\
$\mathrm{~L} / \mathrm{D}_{\text {in }}$ & 4.95 & 5.00 \\
$\mathrm{~h} / \mathrm{L}$ & 0.50 & 0.27 \\
$\gamma$ & 0.16 & 0.10 \\
\hline \hline
\end{tabular}

(b) S-ducts geometrical parameters

Fig. 1 S-duct geometries

\section{B. S-PIV experiment}

A detailed description of the test-rig facility (Fig. 2) and the S-PIV methods was reported by Zachos et al. ${ }^{15}$, and only the key aspects of the experiment are reported here. A bell-mouth intake guides the air through a flowconditioning section which includes a honeycomb mesh to suppress large-scale structures in the flow field. A convergent conical section allows for a smooth transition between the upstream components ( $200 \mathrm{~mm}$ diameter) and the S-duct inlet $\left(\mathrm{D}_{\mathrm{in}}=121.6 \mathrm{~mm}\right)$. At the outlet of the S-duct a borosilicate glass, transparent section permitted optical access for both laser and cameras. A diffuser duct connects the transparent section outlet with a single-stage centrifugal fan which controls the mass-flow rate through the facility. Similar to previous studies in this area ${ }^{11,13,21}$, the intake ducts have no engine face representation to mimic the effects of a fan. Other previous investigations have included a spinner geometry ${ }^{12,14}$, but without any representation of the fan blades. Therefore the potentially 
stabilising effects of the $\operatorname{fan}^{22,23}$ are not accounted for, and the distortions measured in these experiments represent the intrinsic flow instabilities generated within the duct.

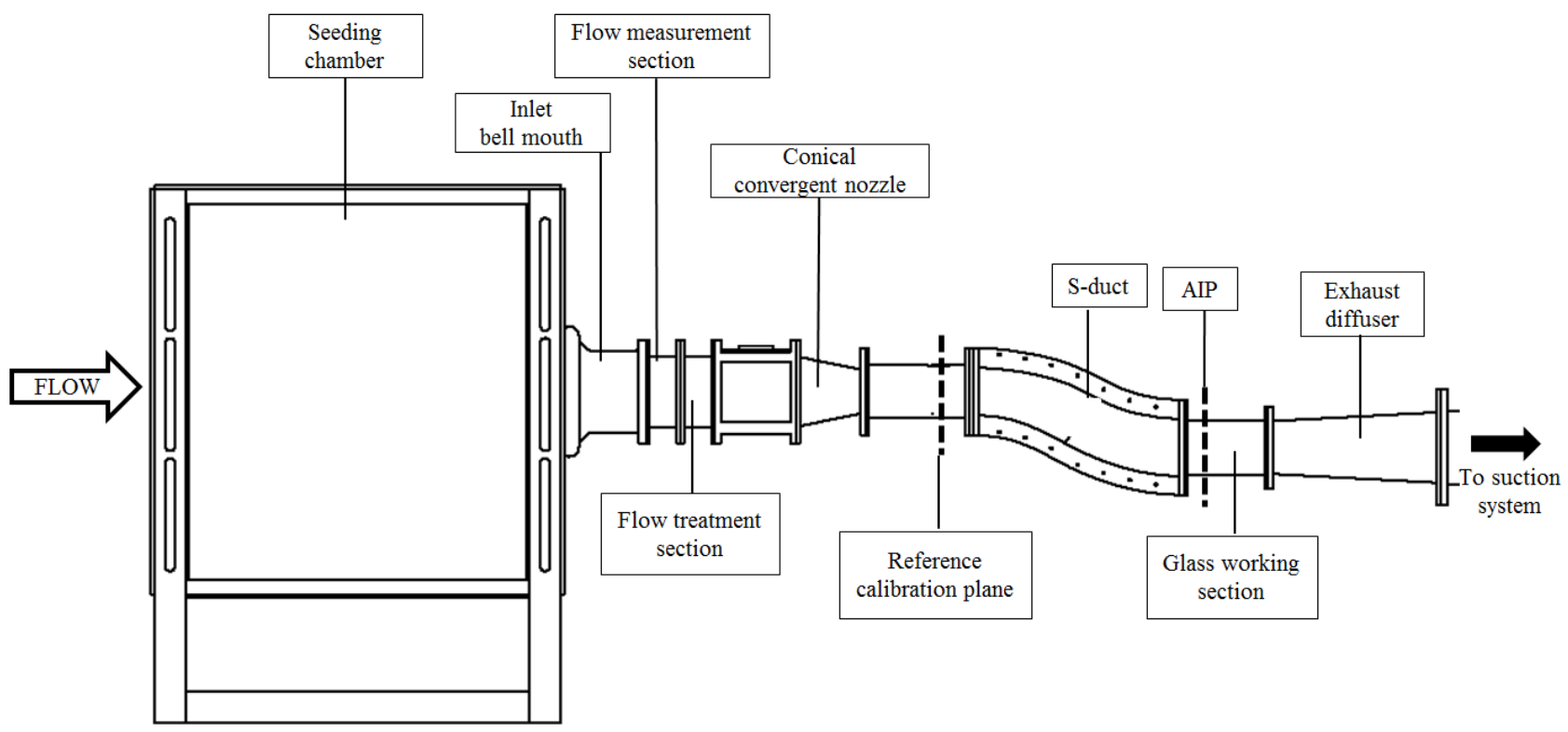

Fig. 2 Test-rig facility ${ }^{15}$

The rig operating condition is kept constant with a control system which acts on the fan rotational speed in order to reach a required value of total-to-static pressure ratio at the flow measurement section (Fig. 2). This pressure ratio is measured with low-bandwidth pressure probes and is previously calibrated with respect to the Mach number at the S-duct reference plane $\left(0.9 \mathrm{D}_{\text {in }}\right.$ upstream the S-duct inlet). Two operating conditions are considered: $\mathrm{M}_{\mathrm{ref}}=0.27$ and $\mathrm{M}_{\text {ref }}=0.60$. The stability of the operating condition depends on the stochastic component of the $\mathrm{M}_{\mathrm{ref}}$ uncertainty, which depends on the random uncertainty of the pressure measurements used to determine the pressure ratio at the flow measurement section. The stochastic $\mathrm{M}_{\mathrm{ref}}$ uncertainty is about $2 \times 10^{-4}$ at both $\mathrm{M}_{\mathrm{ref}}=0.27$ and 0.60 , and is included in the overall $\mathrm{M}_{\text {ref }}$ uncertainty of approximately $0.27 \pm 0.008$ and $0.6 \pm 0.005$ The associated Reynolds numbers based on the inlet diameter, $\mathrm{Re}_{\mathrm{D}}$, are $5.9 \times 10^{5}$ and $13.8 \times 10^{5}$, respectively. The inlet boundary layer at these conditions had a thickness of $\delta=0.07 \mathrm{D}_{\text {in }}$ and $0.06 \mathrm{D}_{\text {in }}$ for $\mathrm{M}_{\text {ref }}=0.27$ and 0.60 respectively. The displacement thickness and shape factor have been calculated using the compressible formulation. The displacement thickness was $\delta^{*}=0.01 \mathrm{D}_{\mathrm{in}}$ and $0.009 \mathrm{D}_{\mathrm{in}}$ for $\mathrm{M}_{\mathrm{ref}}=0.27$ and 0.60 , respectively. At these flow conditions, the shape factors were approximately $\mathrm{H}=1.5$ and 1.7 , respectively. 
The measurements were conducted at the Aerodynamic Interface Plane (AIP) which is located $0.24 \mathrm{D}_{\text {out }}(36 \mathrm{~mm})$ downstream of the actual S-duct outlet. The seeding particles had a diameter of approximately $1 \mu \mathrm{m}$ and were illuminated using a dual cavity, frequency-doubled Nd:YAG laser with a maximum power of $200 \mathrm{~mJ} / \mathrm{pulse}$. The acquisition rate was determined by the laser pulse repetition frequency of $7.5 \mathrm{~Hz}$. TSI InSight 4G software was used to determine the velocity vector field from the S-PIV images. About 9000 velocity vectors were obtained at the AIP which resulted in a spatial resolution of $1.2 \mathrm{~mm}\left(0.008 \mathrm{D}_{\text {out }}\right)$. A disparity correction was applied to account for the possible misalignment between the calibration target and the laser sheet. The analysis of Raffel et al. ${ }^{24}$ was followed to provide an estimate of the overall S-PIV uncertainties, which were approximately $6 \%$ and $8 \%$ for the in-plane and out-of-plane components of the velocity, respectively. Zachos et al. ${ }^{15}$ studied the impact of the number of snapshots on the flow statistics for Duct-2 $(\mathrm{h} / \mathrm{L}=0.27)$ at $\mathrm{M}_{\mathrm{ref}}=0.27$, and concluded that a dataset of 1000 snapshots was adequate to ensure statistical convergence.

\section{Swirl distortion descriptors}

The need to characterise and quantify the distortion patterns presented to the engine has led to the development of swirl distortion descriptors ${ }^{3}$. In this approach, the AIP is divided into several radial rings and the descriptors are evaluated at each radial position based on the ring swirl-angle distribution for each snapshot. A sample twice-perrevolution swirl pattern is shown in Fig. 3 to illustrate the definitions of the swirl distortion descriptors. The swirl angle is assumed positive in the counter-clock wise direction when the AIP is considered from downstream. Positive and negative circumferential extents, $\theta_{i, k}^{+}$and $\theta_{i, k}^{-}$, define the size of the different swirling regions in the i-th ring. Positive and negative sector swirls, $S S_{i, k}^{+}$and $S S_{i, k}^{-}$, are defined as the mean swirl-angle value in each of the swirling regions ${ }^{3}$. 


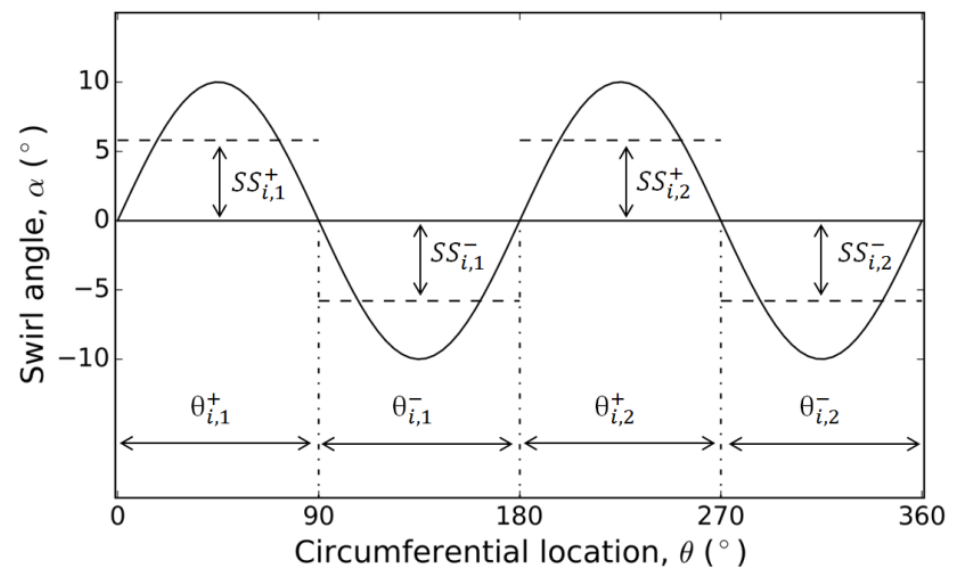

Fig. 3 Twice-per-revolution swirl distortion pattern. Based on the $\mathrm{SAE}^{3}$

Sector swirl and extents are used as the foundation for the development of a range of descriptors which are used to quantify the nature and strength of the swirl distortion. Swirl Intensity (SI) represents the averaged absolute swirl angle in each ring (Eq. 1). Swirl Directivity (SD) represents the overall sense of rotation of the swirling flow in the ring (Eq. 2). Swirl Pairs (SP) indicates the number of swirl pairs relative to the swirl region in the ring which encloses the highest absolute swirl angle content, $\max \left(S S_{i, k}^{+} \theta_{i, k}^{+},\left|S S_{i, k}^{-} \theta_{i, k}^{-}\right|\right)$(Eq. 3). The maximum Sector Swirl in absolute terms and its associated extent in the ring are also considered, and referred to as $\alpha_{1}$ (Eq. 4) and $\theta_{1}$ (Eq. 5), respectively. The descriptors are evaluated at five equal-area rings in order to be consistent with the industry common practice based in conventional pressure probes measurements ${ }^{4}$. At each ring, 72 equi-spaced circumferential points are considered which results in a resolution of $5^{\circ}$. The S-PIV data is linearly interpolated at those locations with the Delaunay triangulation method.

$$
\begin{gathered}
S I(i)=\frac{\sum_{k=1}^{m} S S_{i, k}^{+} \cdot \theta_{i, k}^{+}+\sum_{k=1}^{m}\left|S S_{i, k}^{-}\right| \cdot \theta_{i, k}^{-}}{360} \\
S D(i)=\frac{\sum_{k=1}^{m} S S_{i, k}^{+} \cdot \theta_{i, k}^{+}+\sum_{k=1}^{m} S S_{i, k}^{-} \cdot \theta_{i, k}^{-}}{\sum_{k=1}^{m} S S_{i, k}^{+} \cdot \theta_{i, k}^{+}+\sum_{k=1}^{m}\left|S S_{i, k}^{-}\right| \cdot \theta_{i, k}^{-}} \\
S P(i)=\frac{\sum_{k=1}^{m} S S_{i, k}^{+} \cdot \theta_{i, k}^{+}+\sum_{k=1}^{m}\left|S S_{i, k}^{-}\right| \cdot \theta_{i, k}^{-}}{2 \cdot \operatorname{Max}\left\{S S_{i, k}^{+} \cdot \theta_{i, k}^{+},\left|S S_{i, k}^{-} \cdot \theta_{i, k}^{-}\right|\right\}_{k=1, \ldots, m}} \\
\alpha_{1}(i)=\operatorname{Max}\left\{S S_{i, k}^{+},\left|S S_{i, k}^{-}\right|\right\}_{k=1, \ldots, m} \\
\theta_{1}(i)=\theta_{i, L} \text { where }\left|S S_{i, L}\right|=\alpha_{1}(i)
\end{gathered}
$$




\section{Proper Orthogonal Decomposition}

POD permits the identification of dominant coherent structures present in the flow ${ }^{25}$, and has been recently applied for the analysis of turbulent flows in a wide range of applications, such as the flow in a backward-facing step $^{25,26}$, reciprocating internal combustion engines ${ }^{27}$, turbulent boundary layers ${ }^{28}$ and curved pipes ${ }^{17,20}$. A detailed mathematical description and derivation is provided by Holmes et al. ${ }^{29}$. Considering for simplicity a scalar field $q(x, y, t)$, the POD method finds an orthogonal basis $\left\{\varphi_{j}(x, y)\right\}$ that maximises the projection of $q$ onto $\varphi$. The functions $\varphi_{j}$, often called POD characteristic modes, represent those flow features responsible for most of the signal energy in a statistical sense and may be, sometimes, associated with coherent structures. The POD representation of the original flow field is then obtained by a series of the POD characteristic modes, each of them weighted by a temporal coefficient ${ }^{30}$ (Eq. 6). The temporal coefficients are statistically uncorrelated and the modes are orthonormal. These properties permit the area-averaged mean-squared value of the scalar field to be expressed as a series of the mean-squared value of the temporal coefficients (Eq. 7).

$$
\begin{gathered}
q_{k}(x, y, t)=\sum_{j=1}^{k} a_{j}(t) \varphi_{j}(x, y) \\
\left\langle\overline{{q_{k}}^{2}}\right\rangle=\sum_{j=1}^{k}\left\langle a_{j}{ }^{2}\right\rangle
\end{gathered}
$$

To increase the convergence of the reconstruction, the modal contributions in the series are ordered in decreasing order of the mean-square value of the associated temporal coefficient, which is often referred to as the modal energy. When POD is applied to velocity vector fields, the mean-squared value of the coefficients represents the area-averaged, mean kinetic energy, $\langle\overline{K E}\rangle$, accounted for each mode. The main advantage of the POD reconstruction is its optimality in the sense that, for a given number of terms in the series, the POD maximises the kinetic energy content in the reconstruction ${ }^{31}$. In the present investigation the POD has been implemented with the method of snapshots, developed by Sirovich ${ }^{32}$.

\section{Results}

\section{A. Time averaged and unsteady flow field}

The time-averaged and some unsteady aspects of the AIP flow fields within the high and low offset configurations at $\mathrm{M}_{\mathrm{ref}}=0.27$ and $\mathrm{M}_{\mathrm{ref}}=0.60$ were previously reported by Zachos et al. ${ }^{15}$. Both configurations exhibit the classical low-velocity region in the mean streamwise velocity field at the AIP (Fig. 4a). The higher extent of the 12

American Institute of Aeronautics and Astronautics 
spoiled region for Duct-1 is explained by the greater vertical offset $(\mathrm{h} / \mathrm{L}=0.50)$ which results in stronger secondary flowsh/L. There are notable unsteady aspects of the flow and for both configurations, the maximum out-of-plane velocity fluctuations are observed at the upper bound of the characteristic low-velocity region evident in the mean stream-wise velocity distributions (Fig. 4b). The higher offset configuration (Duct-1) shows a more extensive region of high-fluctuations, with greater peak values which are located in a more central position in the AIP. The Mach number impact on the time-averaged streamwise velocity field $\left(\langle\mathrm{w}\rangle /\left\langle\bar{w}_{A I P}\right\rangle\right)$ is relatively minor ${ }^{15}$. The Mach number does not modify the overall characteristics of the fluctuating stream-wise velocity field $\left(\sigma_{\mathrm{w}} /\left\langle\bar{w}_{A I P}\right\rangle\right)$ while, in general, the maximum levels increase ${ }^{15}$.

The distribution of the time-averaged swirl-angle shows a pair of counter-rotating swirling regions on either side of the symmetry plane (Fig. 5a). These swirling regions are more localised for the low offset configuration (Duct-2) and mostly restricted within the lower sector of the $\mathrm{AIP}^{15}$. The unsteady swirl distributions exhibit notable peak swirl-angle fluctuations which are higher than the mean-flow values for both configurations (Fig. 5b). This highlights the highly unsteady nature of the swirl angle flow field. The Mach number was found to have a modest impact on the time-averaged and fluctuating swirl-angle field ${ }^{15}$. The time-averaged swirl-angle field suggests the presence of the well-known pair of symmetrical, counter-rotating vortices (Fig. 5a), usually referred to in previous investigations ${ }^{11}$. The mean-flow also suggests that the central part of the AIP is relatively swirl-free. However this region is dominated by high swirl-angle fluctuations which can be greater than the mean-flow levels (Fig. 5b). Therefore swirl distortion evaluations based on steady data can result in misleading conclusions about the levels and regions where high-swirl flow distortion occurs ${ }^{15}$.

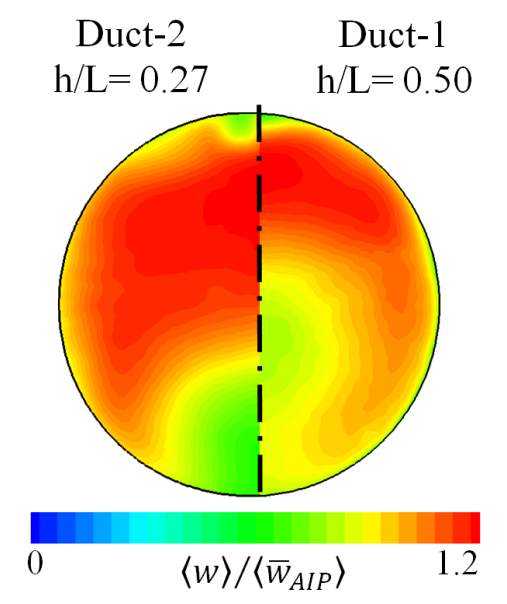

(a) Time-averaged stream-wise velocity

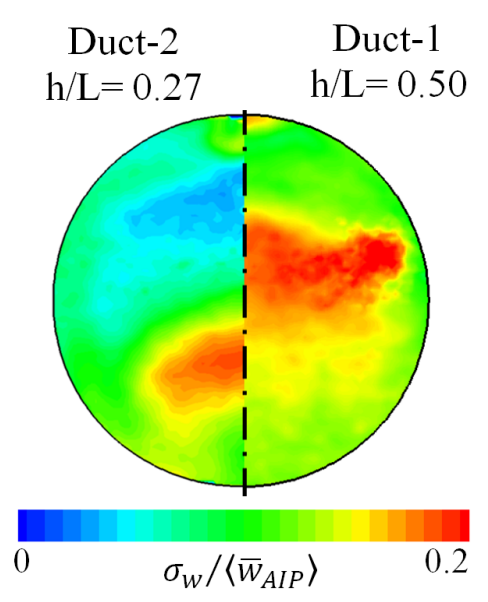

(b) Standard-deviation of the stream-wise velocity

American Institute of Aeronautics and Astronautics 
Fig. 4 Time-averaged and standard-deviation out-of-plane velocity at the AIP for $M_{\text {ref }}=0.27^{15}$

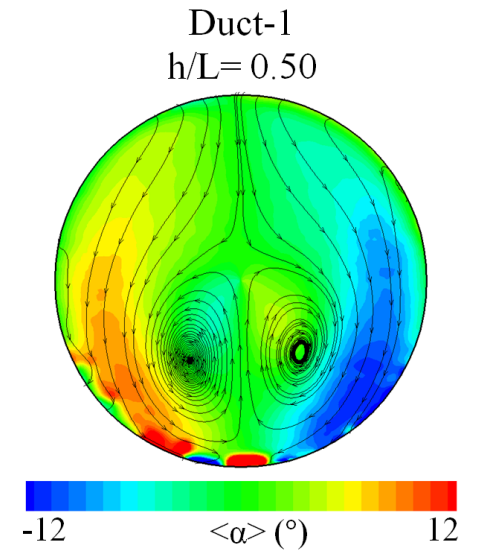

(a) Time-averaged swirl angle

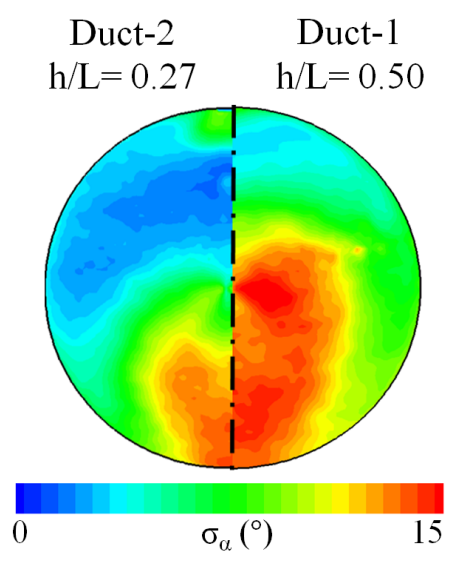

(b) Swirl angle standard deviation

Fig. 5 Time-averaged and standard-deviation swirl-angle at the AIP for $M_{\text {ref }}=0.27^{15}$

\section{B. Radial distribution of swirl distortion}

In this section the radial distribution of unsteady swirl distortion intensity is reported for the first time in these Sduct intakes. Zachos et al. ${ }^{15}$ showed that the impact of the Mach number on the swirl distortion descriptors statistics was minor. Therefore in this work a single Mach number case, $\mathrm{M}_{\mathrm{ref}}=0.27$, is considered for the further in-depth assessment of the swirl distortion. Five equal-area rings are considered with a circumferential resolution of $5^{\circ}$. The data at the outermost ring $(\mathrm{r} / \mathrm{R}=0.95)$ was not used in the analysis since the near-wall region is more prone to be occasionally affected by noise in the S-PIV measurements.

Duct-1 $(\mathrm{h} / \mathrm{L}=0.50)$ shows a monotonic reduction in $\mathrm{SI}_{\text {mean }}$ as the radius increases from $\mathrm{r} / \mathrm{R}=0.32$ to $\mathrm{r} / \mathrm{R}=0.84$ (Fig. 6a). At $\mathrm{r} / \mathrm{R}=0.32 \mathrm{SI}_{\text {mean }}=10.5^{\circ}$, while the value is reduced to $7.9^{\circ}$ at $\mathrm{r} / \mathrm{R}=0.84$. In contrast, Duct- $2(\mathrm{~h} / \mathrm{L}=0.27)$ shows an almost flat distribution of $\mathrm{SI}_{\text {mean }}$, with a constant value of approximately $4.1^{\circ}$. The $\mathrm{SI}$ fluctuations for both ducts decrease monotonically as the radius increases (Fig. 6b). Duct-1 $(\mathrm{h} / \mathrm{L}=0.50)$ shows values of $\mathrm{SI}_{\text {std }}$ of $3.7^{\circ}$ and $1.7^{\circ}$ at $\mathrm{r} / \mathrm{R}=0.32$ and $\mathrm{r} / \mathrm{R}=0.84$, respectively, while the corresponding values for Duct-2 $(\mathrm{h} / \mathrm{L}=0.27)$ are $1.8^{\circ}$ and $0.8^{\circ}$. SI exhibits high fluctuations as reflected by the notable $\mathrm{SI}_{\text {std }}$ values compared with $\mathrm{SI}_{\text {mean }}$. For Duct-1 $\mathrm{SI}_{\text {std }}$ is $35 \%$ and $22 \%$ of $\mathrm{SI}_{\text {mean }}$, for the inner and outer ring respectively, while the corresponding values for Duct- 2 are $44 \%$ and $20 \%$. For both ducts, the highest $\mathrm{SI}_{\max }$ values are encountered at the inner ring and decreases monotonically with the radius (Fig. 6b). The maximum values at the inner ring are approximately $26^{\circ}$ and $17^{\circ}$ for Duct- 1 and Duct-2, 
respectively. This highlights the substantial levels of distortion that can occasionally arise, as these are about 2.5 and 4.1 times the local mean values of swirl angle, respectively.

Conventional measurements and computations are based on steady data obtained using low-bandwidth pressure probes or RANS simulations. To highlight the limitations of steady data it is of interest to compute the SI value obtained from the time-averaged flow field, $\mathrm{SI}_{\text {static }}$ (Fig. 6a). Compared to $\mathrm{SI}_{\text {mean }}, \mathrm{SI}_{\text {static }}$ is lower at all the radial positions for both ducts. For Duct-1, $\mathrm{SI}_{\text {static }}$ corresponds to $19 \%$ and $63 \%$ of the $\mathrm{SI}_{\text {mean }}$ for the inner and outer ring respectively, while for Duct-2 these values are $36 \%$ and $73 \%$. This is because $\mathrm{SI}_{\text {static }}$ does not take into account the fluctuations of the swirl angle flow field, which are averaged out when the mean flow is calculated. Furthermore, while $\mathrm{SI}_{\text {static }}$ suggests the highest swirl values are at the outer-most ring, $\mathrm{SI}_{\text {std }}$ and $\mathrm{SI}_{\max }$ indicates that the highest instantaneous swirl angle levels occur at the inner-most ring. Therefore steady data can lead to misleading conclusions about the swirl distortion levels that occur at the AIP and the radial position where the highest swirl distortion appears. The need to consider the dynamic nature of the distorted flow field highlights the limitations of steady data for swirl distortion assessments, such as those acquired with low-bandwidth swirl probes or from RANS simulations.

. For Duct-1 $(\mathrm{h} / \mathrm{L}=0.50)$ the inner ring shows both the highest $\mathrm{SI}_{\text {mean }}, \mathrm{SI}_{\text {std }}$ and $\mathrm{SI}_{\max }$ values. For Duct-2 $(\mathrm{h} / \mathrm{L}=0.27)$, the $\mathrm{SI}_{\text {mean }}$ is broadly constant across the radius but the inner ring shows the highest $\mathrm{SI}_{\text {std }}$ and $\mathrm{SI}_{\max }$ values (Fig. 6). As the inner ring exhibits the most critical SI levels for both ducts, it is considered in the next sections as the primary region of interest for more detailed investigation of the characteristics of the dynamic distortion. In addition, hub distortion was found to promote the greatest reduction in surge stability margin for the engine studied by Mitchell ${ }^{33}$. However, for completeness, a limited assessment of the swirl distortion unsteady characteristics at the outer ring is also considered. 


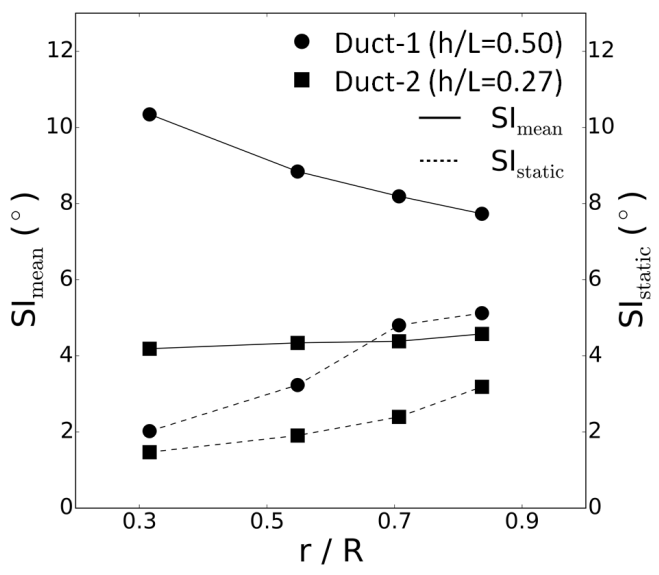

(a) $\mathrm{SI}_{\text {mean }}$ (solid), $\mathrm{SI}_{\text {static }}$ (dashed)

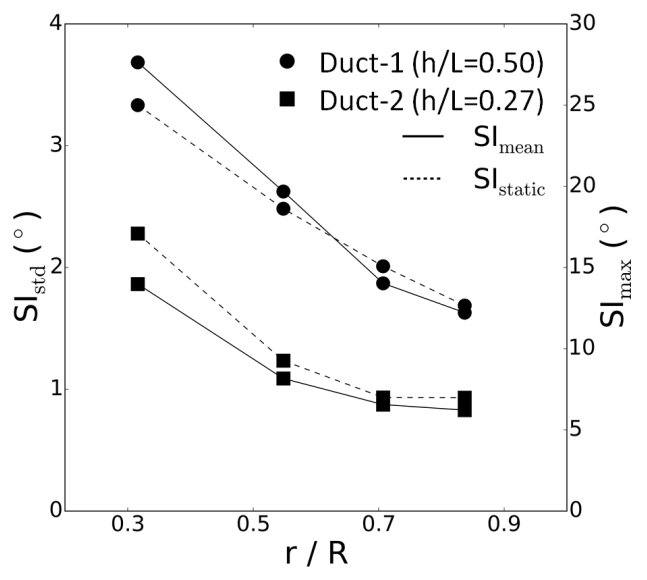

(b) $\mathrm{SI}_{\text {std }}$ (solid), $\mathrm{SI}_{\max }$ (dashed)

Fig. 6 Radial distribution of the Swirl Intensity statistics at $M_{\text {ref }}=0.27$

\section{Swirl Intensity statistical assessment}

In this section, the PDF distribution of the unsteady swirl distortion intensity is analysed for the first time in this kind of intakes. For the calculation of the PDF the range of the SI domain has been discretised into 60 equi-spaced partitions which results in a resolution of approximately $0.5^{\circ}$. The PDFs are then non-dimensionalised (PDF*) with the peak-to-peak value of the SI distribution. As well as the mean and standard deviation, higher moments such as the skewness ${ }^{34}$ and kurtosis ${ }^{35}$ have also been calculated to obtain more insight into the characteristics of the SI distortion.

The statistical characteristics of the SI are evaluated at the inner-most ring $(\mathrm{r} / \mathrm{R}=0.32)$, where the highest $\mathrm{SI}_{\max }$ values are observed (Fig. 6b). The duct offset has a strong impact on the swirl distortion levels as reported by Zachos et al. ${ }^{15}$. For Duct-1 $(\mathrm{h} / \mathrm{L}=0.50), \mathrm{SI}_{\text {mean }}$ and $\mathrm{SI}_{\text {std }}$ at the inner ring $(\mathrm{r} / \mathrm{R}=0.32)$ are about 2.5 and 2 times greater than those of Duct $2(\mathrm{~h} / \mathrm{L}=0.27)$, respectively (Table 1). Relatively high levels of SI skewness are seen for both ducts, especially for Duct-2 (Table 1). This means that extreme swirl distortion events relative to the mean are more likely to occur, as observed in the associated Probability Density Functions (PDFs) (Fig. 7). This is important since, if the turbomachinery design is based on the time-averaged distortion levels, then the peak distortion events which are furthest from the design conditions are the most likely to drive engine instabilities ${ }^{9}$. At $r / R=0.32$, Duct- 1 shows an SI kurtosis of $\mathrm{SI}_{\text {kurt }}=3.6^{\circ}$ which is close to the typical Normal distribution. In contrast, Duct- 2 shows a very high deviation from this value, with a $\mathrm{SI}_{\mathrm{kurt}}$ of $6.9^{\circ}$. This suggests a highly peaked SI distribution around the mean value for Duct-2 (Fig. 7b), while for Duct-1 the SI is more evenly distributed (Fig. 7a). At this inner ring, the SI maximum 
values are $25.0^{\circ}$ and $17.1^{\circ}$ for Duct-1 $(\mathrm{h} / \mathrm{L}=0.50)$ and Duct-2 $(\mathrm{h} / \mathrm{L}=0.27)$ respectively (Table 1$)$. The maximum SI levels are therefore greater in Duct-1 compared to Duct-2. However, note that the $\mathrm{SI}_{\max }$ equates to $6.8 \mathrm{SI}_{\text {std }}$ and 9.2SI $\mathrm{S}_{\text {std }}$ for Duct-1 and Duct-2, respectively. Hence, for Duct-2 the maximum SI event represents a greater deviation compared with the standard deviation of the distribution.

Table 1 Swirl Intensity statistics evaluated at the inner-most $\operatorname{ring}(\mathbf{r} / \mathbf{R}=0.32)$ at $\mathbf{M}_{\mathrm{ref}}=0.27$

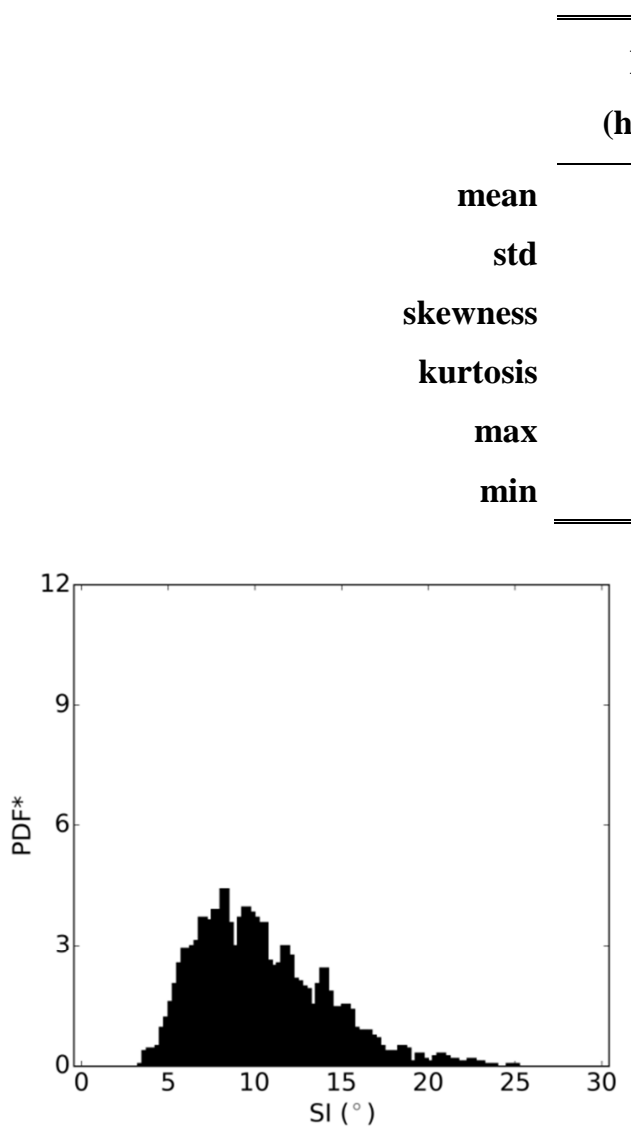

(a) Duct-1 $(\mathrm{h} / \mathrm{L}=0.50)$
Duct-2

Duct-1

$(\mathbf{h} / \mathbf{L}=\mathbf{0 . 2 7})$

$4.2^{\circ}$

$1.9^{\circ}$

1.4

6.9

$17.1^{\circ}$

$1.1^{\circ}$

Fig. 7 Probability Density Function of the SI evaluated at the inner $\operatorname{ring}(r / R=0.32)$ at $M_{\text {ref }}=0.27$

\section{Dynamic swirl distortion characteristics}

Previous investigations on flow distortion in S-ducts ${ }^{14-16}$ have used cloud maps, where instantaneous values of two descriptors are shown together. These maps highlight the range and trend between the descriptors. However, cloud maps do not provide information about the relative frequency of events in each region of the map. In the current study the cloud maps have been replaced by probability maps where the joint-PDF for two descriptors is 
represented. For the evaluation of the joint-PDF, the range of the domain for each of the variables is discretised into 60 equi-spaced partitions. This results in a resolution of approximately $0.5^{\circ}, 0.04$ and 0.04 , for SI, SP and SD, respectively. The joint-PDF is non-dimensionalised $\left(\mathrm{PDF}^{*}\right)$ with the maximum range of the variables. The probability to find the distorted pattern in a certain region of the SP-SD map can be then computed with Eq. $8^{36}$.

$$
P\left(S P_{1}<S P<S P_{2}, S D_{1}<S D<S D_{2}\right)=\int_{S P_{1}}^{S P_{2}} \int_{S D_{1}}^{S D_{2}} P D F d S D d S P
$$

The descriptors are evaluated at the inner-most ring $(\mathrm{r} / \mathrm{R}=0.32)$ where the highest $\mathrm{SI}_{\max }$ values are observed (Fig. 6b). The SP-SD joint-PDF for Duct-1 $(\mathrm{h} / \mathrm{L}=0.50)$ shows a tri-modal behaviour, with three high probability areas around $(\mathrm{SP}=0.5, \mathrm{SD}=-1.0),(\mathrm{SP}=1.0, \mathrm{SD}=0.0)$ and $(\mathrm{SP}=0.5, \mathrm{SD}=-1.0)$ (Fig. 8a). These values correspond to three different swirl distortion patterns: negative bulk swirl, symmetric twin swirl and positive bulk swirl (Fig. 9). To quantify the probability of occurrence of these distortion patterns, the integral of the PDF is calculated over a small region around the corresponding areas in the map. The areas considered to quantify the probability of a negative and positive bulk swirl event are $\mathrm{SP}=[0.5,0.7]$ x $\mathrm{SD}=[-1.0,-0.8]$ and $\mathrm{SP}=[0.5,0.7]$ x $\mathrm{SD}=[0.8,1]$, respectively. For the twin swirl events the area considered is $\mathrm{SP}=[0.9,1.1] \times \mathrm{SD}=[-0.1,0.1]$. For Duct 1 , the estimated probabilities for negative bulk, positive bulk and twin swirl patterns are $11.0 \%, 11.4 \%$ and $9.4 \%$, respectively. Between these states the flow in Duct-1 $(\mathrm{h} / \mathrm{L}=0.50)$ exhibits a wide variety of patterns as suggested by the SP scatter. However it is

possible to observe a high-probability path between these states which follows the expression $S P=\frac{1}{1+|S D|}$. This path represents the lowest value that SP can reach at any value of SD. This relation between SP and SD holds for a oneper-revolution swirl pattern, for which only two swirling regions are present in the swirl angle distribution of the ring, and can be derived from the general formulae (Eq. 2, 3). Accordingly, one of the two swirling regions alternatively becomes dominant $(\mathrm{SP}=0.5, \mathrm{SD}= \pm 1)$ while the other one disappears, so that the flow switches between positive and negative bulk swirl patterns. Amid these two states the two structures eventually become balanced which results in a twin-swirl pattern $(\mathrm{SP}=1, \mathrm{SD}=0)$. This mechanism is referred to as one-per-revolution bulk-totwin switching (Fig. 9). Any deviation from this pattern reflects the presence of additional structures in the ring which therefore results in higher SP values for the same value of SD. 


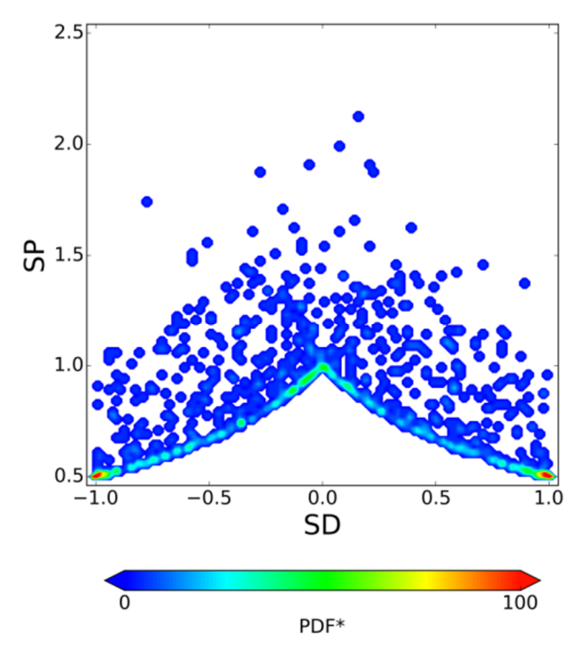

(a) Duct-1 (h/L=0.50)

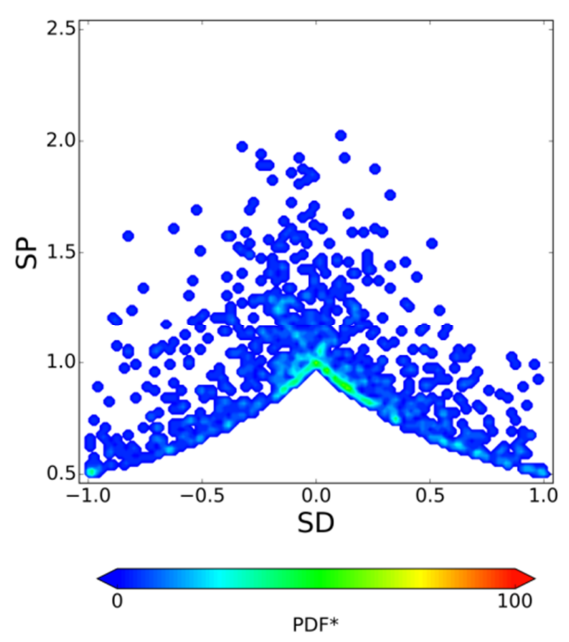

(b) Duct-2 (h/L=0.27)

Fig. 8 SP-SD joint-PDF evaluated at the inner ring $(r / R=0.32)$ at $M_{r e f}=0.27$

The low offset configuration shows a unimodal behaviour at the inner ring $(r / R=0.32)$ with a high-probability region around $(\mathrm{SP}=1.0, \mathrm{SD}=0.0)$ (Fig. 8b). This indicates that the swirl pattern in the inner ring oscillates around twin swirl pattern (Fig. 9). Large deviations from the twin swirl pattern can be observed and even bulk swirl events occur, even though they are less frequent compared to the high offset Duct-1. The probability of a negative bulk, positive bulk and twin swirl event is $3.4 \%, 4.1 \%$ and $14.3 \%$, respectively, considering the same areas as for the high offset duct. Therefore for Duct-2 $(\mathrm{h} / \mathrm{L}=0.27)$ twin swirl patterns occur more frequently compared to Duct-1 ( $h / L=0.50)$, while bulk-swirl events are significantly less likely to appear. Furthermore Duct-2 $(\mathrm{h} / \mathrm{L}=0.27)$ does not show particularly high-PDF levels upon the one-per-revolution bulk-to-twin switching path, as opposed to Duct-1 $(\mathrm{h} / \mathrm{L}=0.50)$, and the bulk swirl patterns appear as more isolated events. 


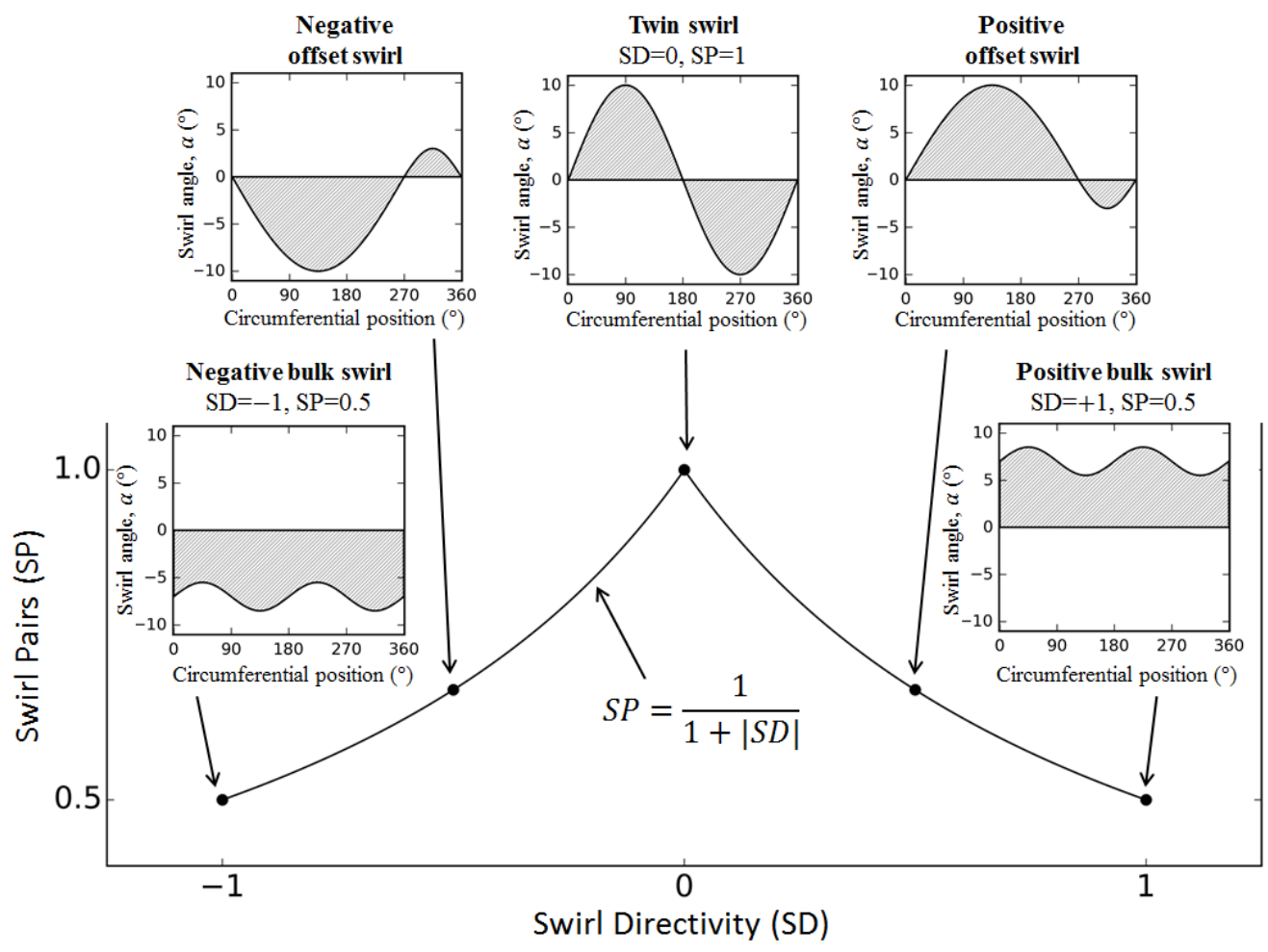

Fig. 9 One-per-revolution bulk-to-twin swirl switching

It is of interest to identify which swirl pattern is responsible for the highest SI events. For both ducts the general trend at the inner ring $(\mathrm{r} / \mathrm{R}=0.32)$ is for the SI to increase as SP decreases, even though the scatter in SI for a given SP is significant (Fig. 10). For example, in the high offset Duct-1 it is possible to find values of SI which range between $5^{\circ}$ and $25^{\circ}$ for a given $\mathrm{SP}=0.5$ (Fig. 10a). For both configurations the bulk swirl patterns ( $\left.\mathrm{SP}=0.5\right)$ are associated with the most intense distortion events in terms of SI (Fig. 10). Bulk swirl events are more common for the high offset Duct-1 $(\mathrm{h} / \mathrm{L}=0.50)$ compared with Duct-2 $(\mathrm{h} / \mathrm{L}=0.27)$ (Fig. 8). That is the reason for the higher number of peak SI events in the high offset Duct-1, while peak SI events appear as more isolated events for the low offset Duct-2 (Fig. 7). For both configurations SP values as high as 2.0 are promoted even though the likeliness of occurrence is low (Fig. 10). These high SP events are associated with low SI values, with values between approximately $4^{\circ}-10^{\circ}$ and $2^{\circ}-4^{\circ}$ for Duct- $1(\mathrm{~h} / \mathrm{L}=0.50)$ and Duct- $2(\mathrm{~h} / \mathrm{L}=0.27)$, respectively. 


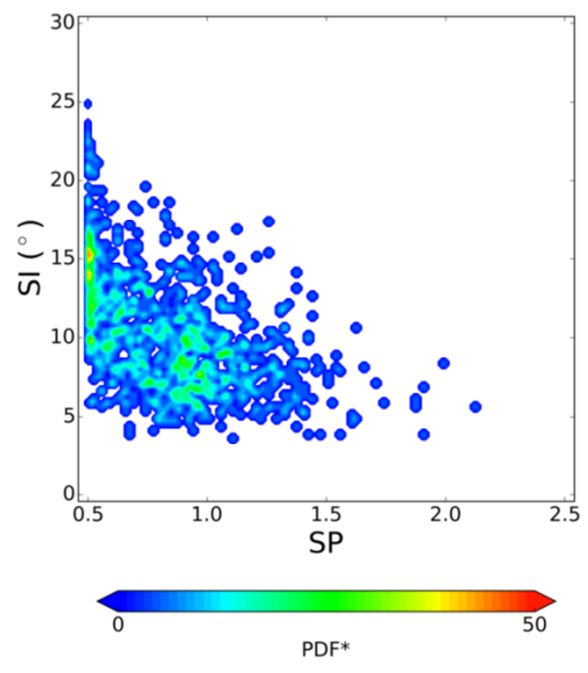

(a) Duct-1 $(\mathrm{h} / \mathrm{L}=0.50)$

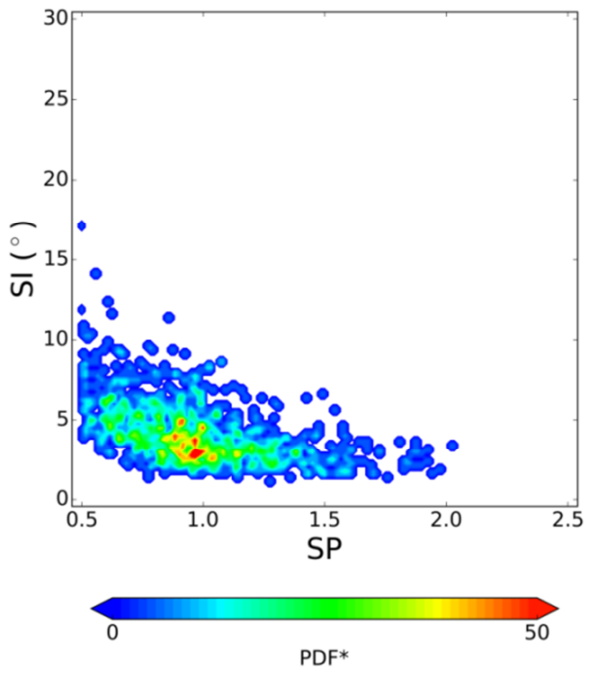

(b) Duct-2 $(\mathrm{h} / \mathrm{L}=0.27)$

Fig. 10 SI-SP joint-PDF evaluated at the inner ring $(r / R=0.32)$ at $M_{r e f}=0.27$

At the inner ring $(\mathrm{r} / \mathrm{R}=0.32)$, for both ducts the greatest $\mathrm{SI}$ values are typically associated with bulk swirl patterns $(\mathrm{SP}=0.5)$ (Fig. 10). The signature is not as clear in the outer ring $(\mathrm{r} / \mathrm{R}=0.84)$, where the peak SI values are associated with a wider range of SP values for both configurations (Fig. 11). The greatest SI values in Duct-1 (h/L=0.50) are associated with SP values between approximately 0.5 and 1.2 (Fig. 11a) with the relatively high values of PDF* centered around $\mathrm{SP}=0.8$. For Duct-2 there is very little difference in the peak SI values across almost all the range of SP and the relatively high $\mathrm{PDF}^{*}$ are similarly broadly spread across about SP=0.9 to 1.5 (Fig. 11b). Along with the generally lower values of SI associated with a broader range of SP, at the outer ring there is a slightly greater peak value of SP 2.5 relative to the levels of about 2.0 observed for the inner ring. Overall, the distortion signature of the outer ring indicates lower levels of SI across a broader range of SP. It is expected that this is partially because the swirl distortion metrics in the outer region are affected by additional, smaller flow features and secondary flows which predominately arise in the regions near to the outer wall. 


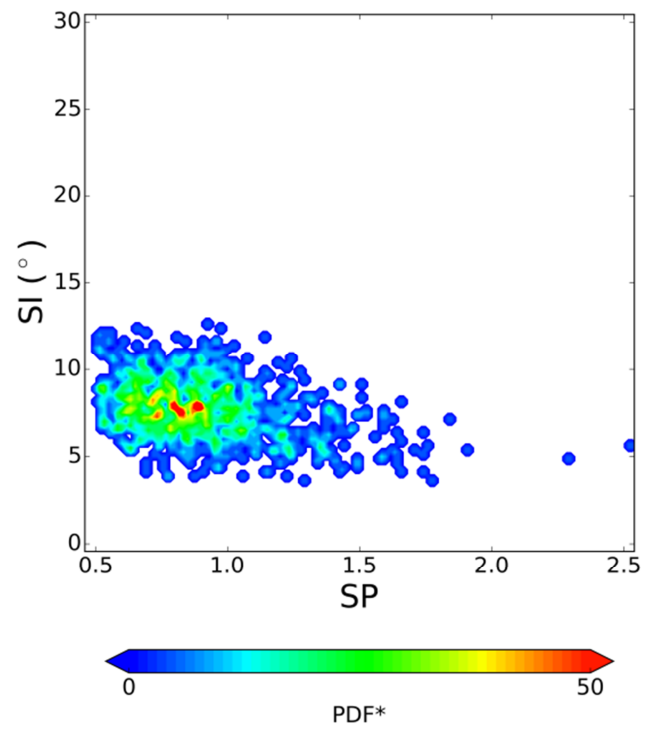

(a) Duct-1 $(\mathrm{h} / \mathrm{L}=0.50)$

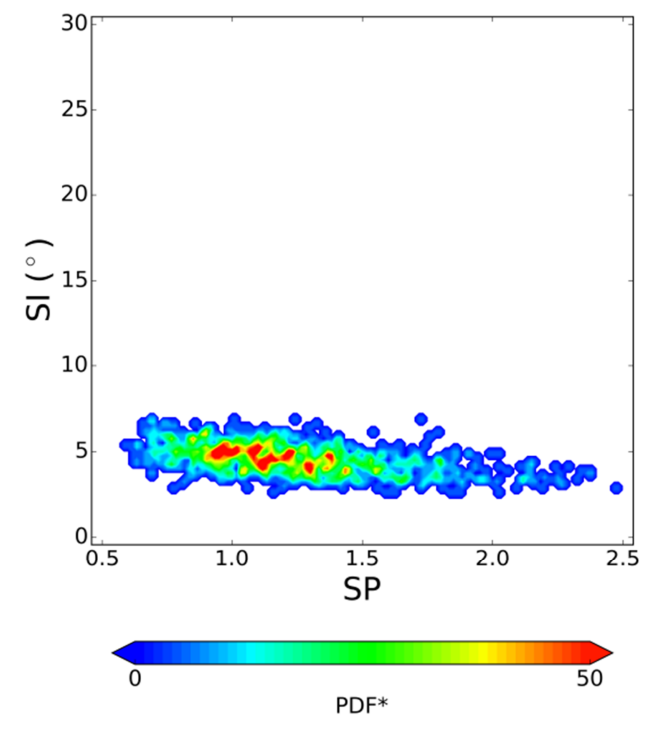

(b) Duct-2 (h/L=0.27)

Fig. 11 SI-SP joint-PDF evaluated at the outer ring $(r / R=0.84)$ at $M_{r e f}=0.27$

\section{E. High-swirl, reduced-extent events}

The SI parameter is defined as the absolute swirl angle over the whole extent of the individual ring (Eq. 1). Therefore this descriptor provides a representative value of the swirl angle within the spoiled region when the different swirling regions show similar swirl sector values (Fig. 3). However when the swirl sector is greater in one particular region of relatively reduced extent, the SI formulation returns a value that can be substantially lower than the actual swirl angle in the most spoiled region (Fig. 12). To highlight these high-swirl, reduced-extent distortion patterns the $\alpha_{l}$ and $\theta_{1}$ descriptors are applied. The $\alpha_{l}$ and $\theta_{1}$ parameters are defined as the sector swirl and extent of the swirling region associated with the highest sector swirl in absolute terms (Eq. 4, 5). By definition $\alpha_{1} \geq S I$, and $\alpha_{1}=S I$ only when all the swirling regions in the ring show the same sector swirl value. Particular cases of this situation are the bulk-swirl and twin-swirl patterns. High-swirl events with a partial extent can trigger instabilities if the circumferential extent of the spoiled region, $\theta_{1}$, exceeds a critical value. As a guide for the estimation of the critical angle Cousins ${ }^{8}$ suggests the extent covered by 4 or 5 blades in a typical axial stage. Accordingly, for a typical fan rotor stage with $20-24$ blades the critical angle would be in the range of $60^{\circ}-90^{\circ}$. 


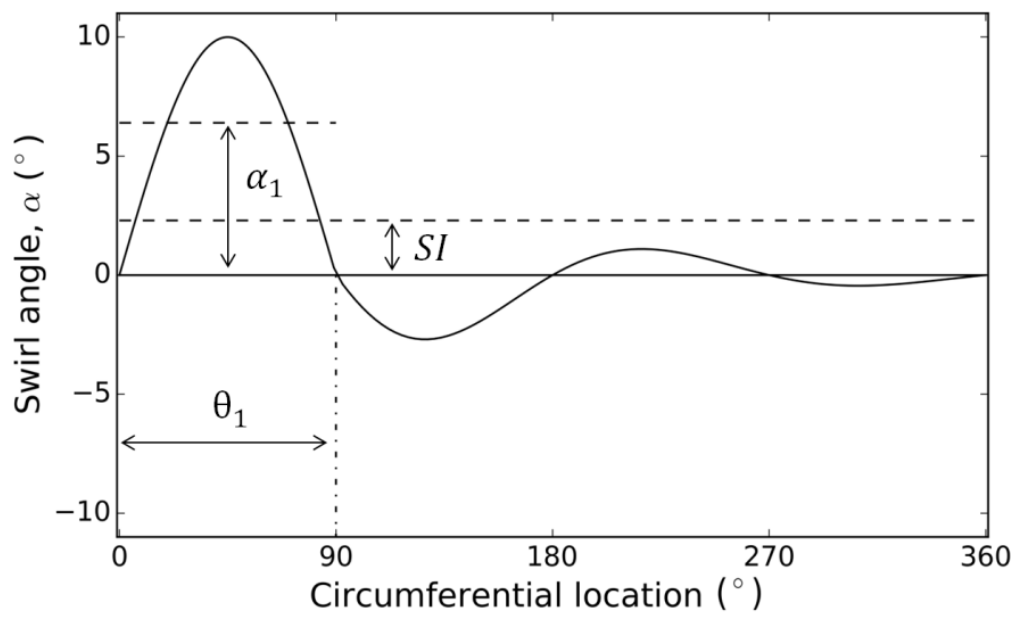

Fig. 12 Swirl distortion pattern for which SI is not representative of the swirl level in the most spoiled region

For Duct-1 $(\mathrm{h} / \mathrm{L}=0.50)$ high values of $\alpha_{1}$ occur for all the range of $\theta_{1}$ (Fig. 13a). Those events associated with $\theta_{1}=360^{\circ}$ correspond by definition to bulk swirl patterns, for which $\alpha_{1}=$ SI. Therefore for these events both $\alpha_{1}$ and SI provide the same information. However for smaller extents $\theta_{1}$ there are high values of $\alpha_{1}$ associated with substantially lower SI. These events correspond to swirl patterns in which one of the sector swirls show greater swirl angle levels compared to the rest of the swirling regions in the ring (Fig. 12). The greater the difference between the dominant sector swirl compared with the others, the greater is the difference between $\alpha_{1}$ and SI. For Duct-1 ( $\mathrm{h} / \mathrm{L}=0.50$ ), the difference between SI and $\alpha_{1}$ is notable for the large $\alpha_{1}$ events associated with extents lower than $200^{\circ}$ (Fig. 13a). For example, there are values of $\alpha_{1}$ greater than $25^{\circ}$ associated with extents between $60^{\circ}-200^{\circ}$, for which SI show values between $6^{\circ}-13^{\circ}$ lower than $\alpha_{1}$. Those high- $\alpha_{1}$ events associated with extents $\theta_{1}$ greater than the estimated critical angle of $60^{\circ}-90^{\circ}$ could adversely affect the stability of the engine and should be considered for the intake-engine compatibility assessment. The SI descriptor is not an appropriate metric to identify these potent, highswirl events with a reduced extent, for which $\alpha_{1}$ and $\theta_{1}$ are shown to be more useful.

For Duct-2 $(\mathrm{h} / \mathrm{L}=0.27)$ there are also high $\alpha_{1}$ values associated with extents $\theta_{1}$ between $60^{\circ}-140^{\circ}$, for which the SI is substantially lower than $\alpha_{1}$ (Fig. 13b). These events show values of $\alpha_{1}$ around $20^{\circ}$, while the corresponding SI levels are between $6^{\circ}-9^{\circ}$. The low offset Duct- 2 shows peak $\alpha_{1}$ values which are lower compared with the high offset Duct-1. The peak $\alpha_{1}$ values are $22^{\circ}$ and $27^{\circ}$ for Duct-2 and Duct-1, respectively. Moreover for Duct-2 $(\mathrm{h} / \mathrm{L}=0.27)$ these events with large $\alpha_{1}$ and reduced-extent are mostly associated with $\theta_{1}$ confined between $60^{\circ}-140^{\circ}$. In contrast, for the high offset Duct-1 high- $\alpha_{1}$ events occur for all the range of $\theta_{1}$. The SI descriptor value is affected 
by the extent of the swirling region. The two duct configurations show different $\theta_{1}$ signatures and therefore it is proposed that a more useful assessment of the potency of the individual swirl distortions can be done with $\alpha_{1}$ and $\theta_{1}$.

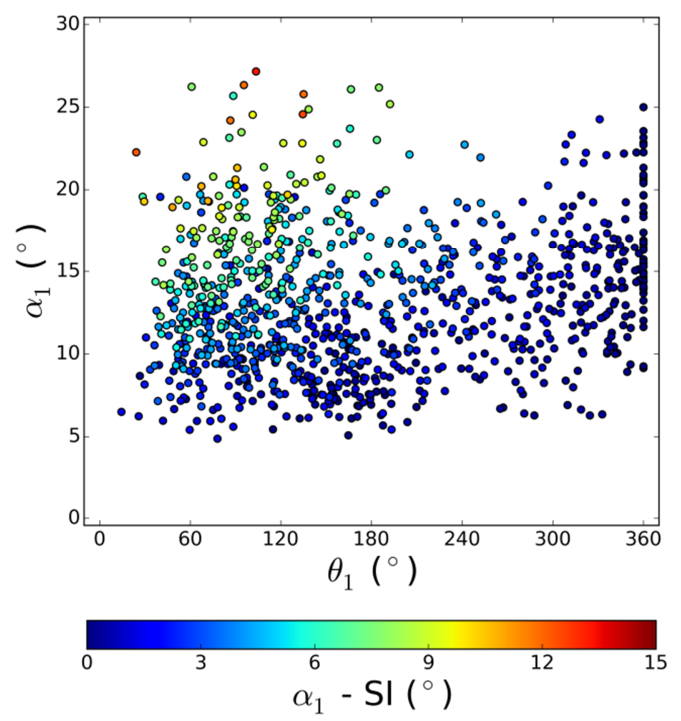

(a) Duct-1 $(\mathrm{h} / \mathrm{L}=0.50)$

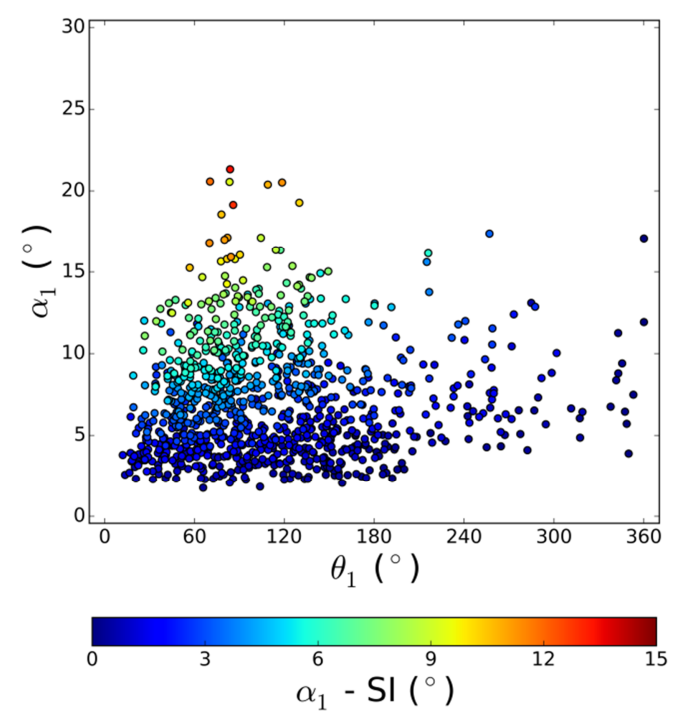

(b) Duct-2 (h/L=0.27)

Fig. $13 \alpha_{1}-\theta_{1}-S I$ cloud maps evaluated at the inner ring $(r / R=0.32)$ at $M_{r e f}=0.27$

\section{F. Proper Orthogonal Decomposition at the AIP}

Results discussed in the previous sections suggest the presence of different flow distortion modes for each duct. To shed light on the most energetic coherent structures present in the flow field at the outlet of S-duct intakes, the Proper Orthogonal Decomposition is applied to the velocity vector at the AIP. To the authors' knowledge this is the first attempt to apply POD on measured three-component velocity vectors at the AIP of S-duct intakes. The aim of the POD analysis is to understand the flow modes that affect the unsteady flow field and the distortion metrics. Mode 0 represents the mean flow and is referred to as Mean-Flow Mode (MFM), while the rest of the modes are interpreted as perturbations over the mean ${ }^{37}$. The temporal coefficient associated with the MFM shows a high mean value with negligible oscillations. The temporal coefficients associated with the other modes oscillate around the null mean-value. The modes are ordered by $\langle\overline{T K E}\rangle$ content.

\section{The effect of duct offset $(h / L)$}

The $\langle\overline{K E}\rangle$ accounted for by the mean-flow (MFM) is $93.3 \%$ and $97.5 \%$ for Duct-1 $(\mathrm{h} / \mathrm{L}=0.50)$ and Duct-2 ( $\mathrm{h} / \mathrm{L}=0.27$ ), respectively. The fraction of energy the mean flow contributes to $\langle\overline{K E}\rangle$ is indicative of the complexity of 
the flow ${ }^{25}$. The rest of the mean energy is accounted for by the turbulent fluctuations $\langle\overline{T K E}\rangle$ promoted by perturbations represented by the rest of POD modes. Even though the turbulent energy represents a much lower fraction of the overall mean-energy in comparison to the mean flow, these fluctuations are of prime importance since they are responsible for the peak distortion events. The larger $\langle\overline{T K E}\rangle$ contribution comes from the first two modes, which cumulatively account for $26 \%$ and $20 \%$ of the overall $\langle\overline{T K E}\rangle$ for Duct-1 $(\mathrm{h} / \mathrm{L}=0.50)$ and Duct-2 $(\mathrm{h} / \mathrm{L}=0.27)$ respectively (Fig. 14). The rest of the $\langle\overline{T K E}\rangle$ is accounted for by the higher-order modes. However the energy content reduces relatively slowly which indicates that a large number of modes need to be considered to capture the overall energy of the flow field. The effect of the inlet Mach number on the modal energy spectrum is modest (Fig. 14). The first two modes account for $26 \%$ of the $\langle\overline{T K E}\rangle$ for Duct-1 $(\mathrm{h} / \mathrm{L}=0.50)$ at both $\mathrm{M}_{\mathrm{ref}}=0.27$ and 0.60. For Duct-2 $(\mathrm{h} / \mathrm{L}=0.27)$ these values are $20 \%$ and $16 \%$. About the mean-flow contribution to the area-averaged, time-averaged kinetic energy, $\langle\overline{K E}\rangle$, Duct-1 $(\mathrm{h} / \mathrm{L}=0.50)$ values at $\mathrm{M}_{\mathrm{ref}}=0.27$ and 0.60 are $93.3 \%$ and $92.4 \%$, respectively. The corresponding values for Duct-2 $(\mathrm{h} / \mathrm{L}=0.27)$ are $97.5 \%$ and $95.5 \%$. For both configurations, the inlet Mach number does not alter the most-energetic, fundamental structures of the velocity field. The small impact of the inlet Mach number on the POD modal shapes was previously observed by MacManus et al. ${ }^{16}$ for the DDES of the total pressure field in the same non-dimensional geometries studied in this work.

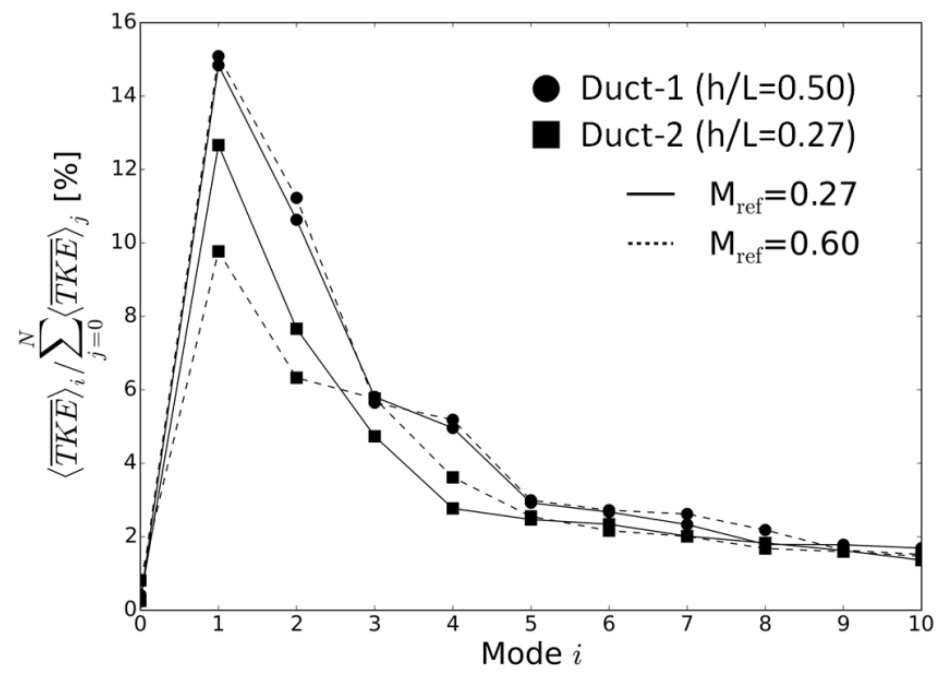

Fig. 14 POD modal energy distribution

For the low offset Duct $2(\mathrm{~h} / \mathrm{L}=0.27)$ the first two most-energetic modes show distinct characteristics which influence the nature of the unsteady perturbations (Fig. 15). Mode 1 predominately represents a perturbation of the vertical velocity field in the lower sector of the AIP (Fig. 15b), and is referred to as Vertical Mode (VM). The VM 25 American Institute of Aeronautics and Astronautics 
shows a symmetric distribution for the stream-wise velocity component with respect to the vertical symmetry plane (Fig. 15a), which indicates a modulation of the vertical extent of the low stream-wise velocity region around its mean position. The effect of the VM on the mean flow is illustrated in Fig. 16a, b, where snapshots of the flow field are shown when only the contribution of the mean flow and VM are considered. The minimum and maximum values of $a_{V M}(\mathrm{t})$ are considered to illustrate the greatest impact of this modal structure. When $a_{V M}(\mathrm{t})=0$ the reconstructed flow is simply the mean flow. When the associated temporal coefficient $a_{V M}(\mathrm{t})$ is positive the main loss region extends upwards (Fig. 16a), while the opposite occurs for negative $a_{V M}(\mathrm{t})$ values for which the spoiled region becomes more confined to the lower wall (Fig. 16b). The central region of the stream-wise component of VM (Fig. 15a) is associated with the modulation of the spoiled region vertical extent. This area is mainly co-incident with the upper part of the mean stream-wise velocity deficit and the area of maximum $\sigma_{w}$ (Fig. 4). Consequently the vertical perturbation is postulated to be associated with the unsteadiness of the shear layer, which is linked to the classic diffusion and stream-wise separation at the inner bend of the S-duct. The in-plane streamlines of VM (Fig. 15b) show a symmetric pair of vortices at each side of the vertical symmetry plane. This pattern is qualitatively similar to the mean-flow distribution, even though the vortices are located further from the symmetry plane. The effect on the mean flow is to modify the strength, position and area covered by the vortex pair. When $a_{V M}(\mathrm{t})$ is positive the pair of vortices becomes stronger (Fig. 16a), whereas when $a_{V M}(\mathrm{t})$ is negative they are confined towards the lower wall region. For a sufficiently low value of $a_{V M}(\mathrm{t})$ the vortices eventually do not arise and the in-plane topology is simply dominated by the general downward pitching flow expected at the exit of the duct (Fig. 16b).

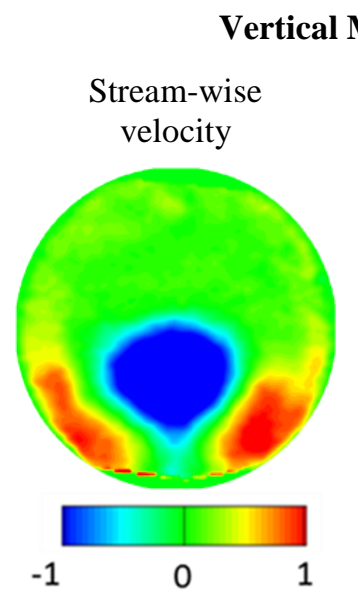

(a) $\Phi_{V M}^{w}$

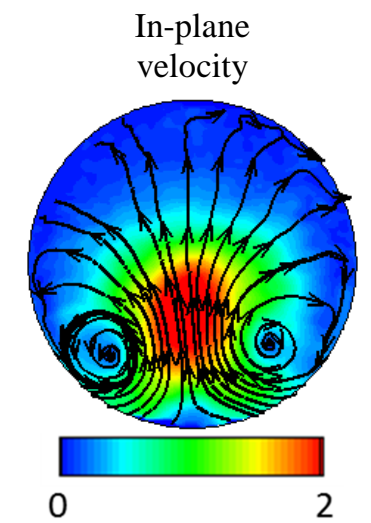

(b) $\Phi_{V M}^{V_{i p}}$

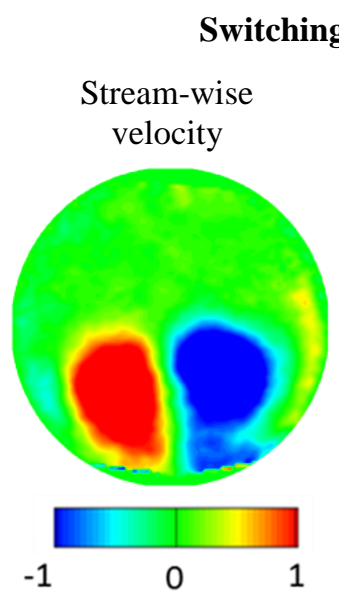

(c) $\Phi_{S M}^{w}$

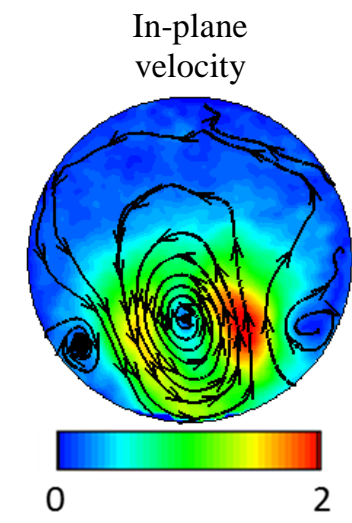

(d) $\Phi_{S M}^{V_{i p}}$

Fig. 15 POD modal shapes for Duct-2 $(h / L=0.27)$ at $M_{\text {ref }}=0.27$ 
Mode 2 shows a dominant swirling cell as revealed by the perturbation of the in-plane velocity field and the associated streamlines pattern (Fig. 15d). This perturbation of the in-plane velocity components was previously observed downstream of simpler, non-diffusing pipes with a single $90^{\circ}$ bend by Hellström et al. ${ }^{20}$ and Kalpakli Vester et al. ${ }^{17}$, and referred to as Switching Mode (SM). This title was chosen since this perturbation is responsible for the switching mechanism by which alternately one of the vortices observed in the mean-flow becomes dominant. Depending on the sign of $a_{S M}(\mathrm{t})$ the swirling cell perturbation rotates in either clock or anti-clock wise direction. The swirl switching mechanism is evident in the snapshots of the flow field reconstructed using only the SM superimposed on the mean flow field (Fig. 16c,d). The extreme values of $a_{S M}(\mathrm{t})$ are considered to show the maximum deviation from the symmetric pair of vortices observed in the mean flow. The Switching Mode is not energetic enough to promote a single rotating cell in the AIP. That is reflected by the fact that although the mode does result in a switching characteristic, $\left|a_{S M}(\mathrm{t})\right|$ does not reach high enough values to completely bias the flow field in one rotating direction. The SM shows an antisymmetric distribution of the stream-wise velocity component with respect to the vertical symmetry plane (Fig. 15c). This indicates a modulation of the main loss region, so that its position oscillates circumferentially with the swirl switching. For example, when the left vortex dominates the AIP flow field (Fig. 16c), it migrates towards a more centred position while confining the other vortex towards the wall. The primary loss region then follows the movement of the dominant vortex.

\section{Vertical Mode (VM)}

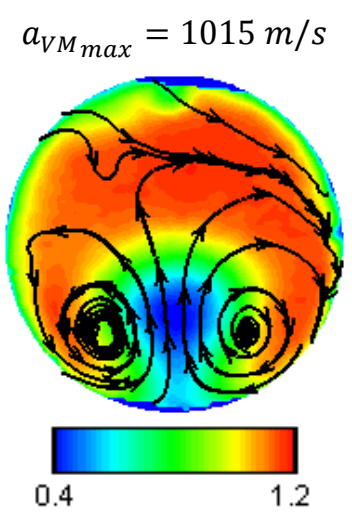

(a) $w_{M F+V M} /\left\langle\bar{w}_{A I P}\right\rangle$

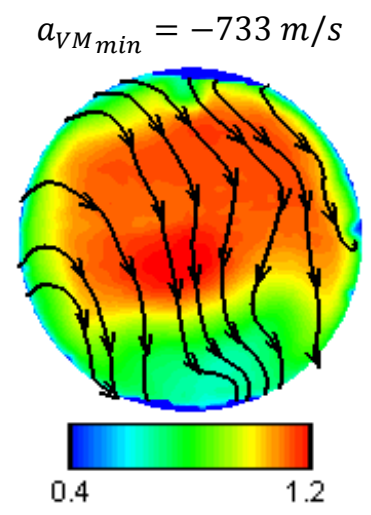

(b) $w_{M F+V M} /\left\langle\bar{w}_{A I P}\right\rangle$

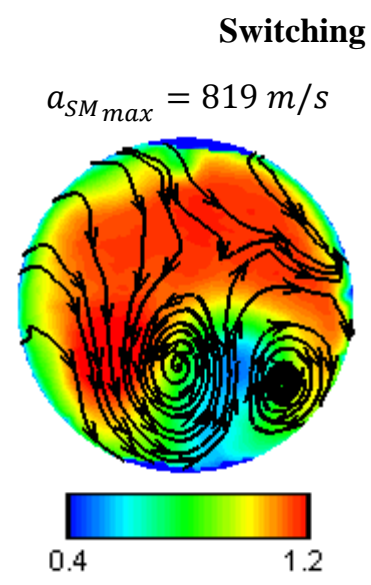

(c) $w_{M F+S M} /\left\langle\bar{w}_{A I P}\right\rangle$

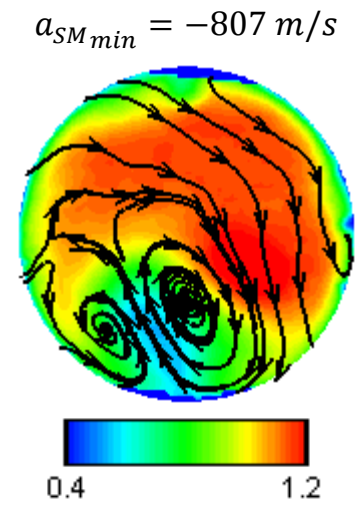

(d) $w_{M F+S M} /\left\langle\bar{w}_{A I P}\right\rangle$

Fig. 16 Snapshots for the Vertical and Switching Modes super-imposed on the mean-flow (Duct-2, $h / L=0.27$,

$$
\mathbf{M}_{\text {ref }}=\mathbf{0 . 2 7 )}
$$


Duct-1 $(\mathrm{h} / \mathrm{L}=0.50)$ shows the same qualitative modal shapes which correspond to the Vertical and Switching Modes (Fig. 17), even though some topological differences are noted such as the different position and extent of the flow features. In addition, the energetic content of the Vertical and Switching Modes is changed, so that the switching mode becomes the dominant unsteady structure in the flow within Duct-1. The $\langle\overline{T K E}\rangle$ content for Duct-1 $(\mathrm{h} / \mathrm{L}=0.50)$ is $11 \%$ and $15 \%$ for VM and SM, respectively. The corresponding values for Duct-2 $(\mathrm{h} / \mathrm{L}=0.27)$ are $13 \%$ and $8 \%$. As opposed to the low offset Duct-2 ( $\mathrm{h} / \mathrm{L}=0.27)$, for the high offset Duct-1 the temporal coefficient associated with the Switching Mode, $a_{S M}(\mathrm{t})$, reaches absolute-values high enough to promote both positive and negative single rotating cells (Fig. 18). The effect of the Vertical Mode on the mean flow (MFM) is very similar to that in Duct-2, previously illustrated in Fig. 16a,b.

MacManus et al. ${ }^{16}$ studied the spectral content of the most-energetic coherent structures for two S-ducts with the same non-dimensional geometries as Duct-1 and Duct-2, at a slightly larger geometric scale of 1.08. The POD was applied on the DDES of the total pressure field simulated at an inlet Mach number of 0.28 . The two most-energetic modes for the total pressure field were similar to the stream-wise velocity perturbations promoted by the Switching and Vertical Modes observed in the current measurements. The Switching Mode was associated with a frequency of $\mathrm{St}=0.55$ and $\mathrm{St}=0.4$ for the high and low offset configurations, respectively. The dominant frequency for the Vertical Mode was $\mathrm{St}=0.90$ and $\mathrm{St}=0.75$, for the high and low offset ducts respectively ${ }^{16}$.

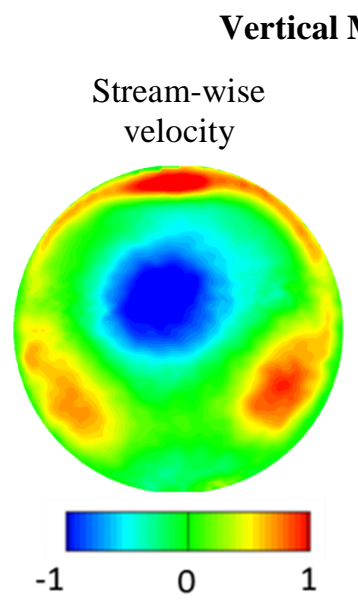

(a) $\Phi_{V M}^{W}$

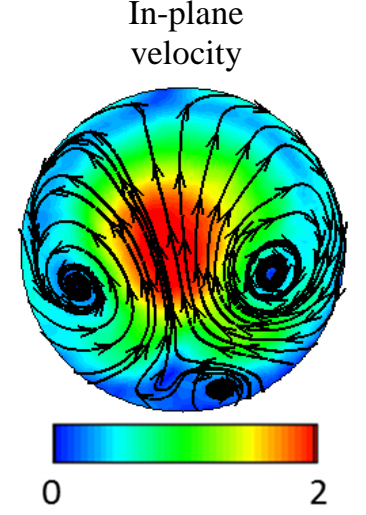

(b) $\Phi_{V M}^{V_{i p}}$

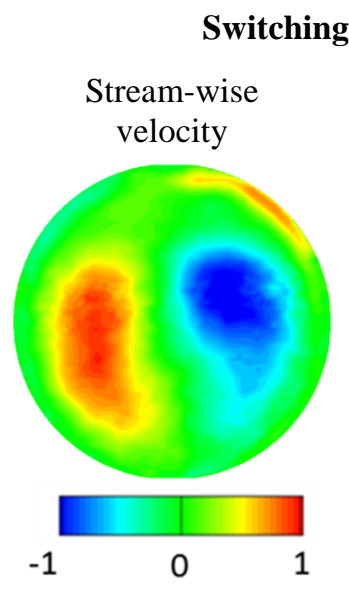

(c) $\Phi_{S M}^{w}$

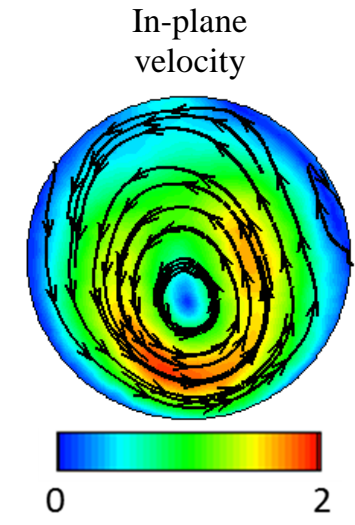

(d) $\Phi_{S M}^{V_{i p}}$

Fig. 17 POD modal shapes for Duct-1 $(\mathrm{h} / \mathrm{L}=0.50)$ at $\mathrm{M}_{\mathrm{ref}}=0.27$ 
Switching Mode (SM)

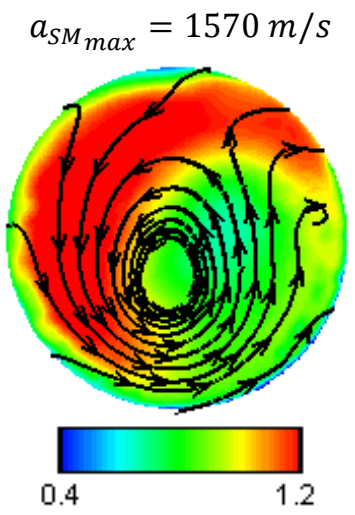

(a) $w_{M F+S M} /\left\langle\bar{w}_{A I P}\right\rangle$

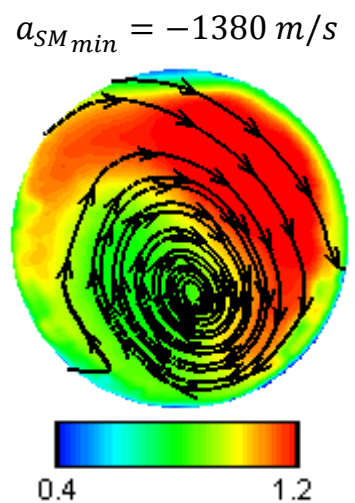

(b) $w_{M F+S M} /\left\langle\bar{w}_{A I P}\right\rangle$

Fig. 18 Snapshots for the Switching Mode super-imposed on the mean-flow (Duct-1, $h / L=0.50, M_{r e f}=0.27$ )

The POD of the three components of the velocity vector measured with S-PIV permits the combined investigation of both the stream-wise and in-plane flow characteristics. This allows for a better understanding of the three dimensional flow features associated with the main loss mechanisms, which are usually investigated based on total pressure data. For example, Garnier ${ }^{14}$ used high-bandwidth transducers to measure the total pressure field at the outlet of an S-duct with the same non-dimensional geometry as Duct-1 $(\mathrm{h} / \mathrm{L}=0.50)$. Garnier reported a strong, outof-phase coherence at $\mathrm{St}=0.48$ between the unsteady total pressure measured within the high fluctuation region on both sides of the symmetry plane, for a flow condition of $\mathrm{M}_{\mathrm{AIP}}=0.20$. The stream-wise perturbation promoted by the Switching Mode observed in the current measurements (Fig. 17c) can explain the out-of-phase coherence of total pressure for two points located at both sides of the symmetry plane. In addition the DDES simulation performed by MacManus et al. ${ }^{16}$ associated this switching perturbation with a frequency of $\mathrm{St}=0.55$ which is similar to the $\mathrm{St}=0.48$ observed by Garnier ${ }^{14}$, for the same geometry and flow conditions. The POD of the full velocity vector presented in this work reveals the three dimensional characteristics of this flow perturbation, which is associated with the swirl switching (Fig. 17d). The POD of the velocity vector could also provide a better understanding of the flow mechanisms which promote both total pressure and swirl distortion. Total pressure distortion is mostly governed by the stream-wise velocity non-uniformities, while swirl distortion is related to both stream-wise and in-plane velocity fields. Even though in S-ducts the regions of total pressure distortion are usually associated with high levels of swirling flow ${ }^{3}$, both phenomena are typically investigated separately ${ }^{7,14,38}$. There is a lack of knowledge about the interaction between total pressure and swirl distortion ${ }^{3}$. The present investigation shows that the most-energetic flow 
mechanisms in S-ducts promote both stream-wise and in-plane perturbations. The study of both stream-wise and inplane velocity characteristics has the potential to enhance the knowledge of the relation between total-pressure and swirl distortion.

\section{Modes impact on distortion metrics}

The highly dynamic behaviour of the swirl distortion metrics has been demonstrated for the two configurations under investigation (Fig. 8). The analysis has shown that the distorted swirl pattern in the inner ring $(\mathrm{r} / \mathrm{R}=0.32)$ deviates from the mean-flow twin swirl towards bulk swirl distributions which promote the highest SI levels (Fig. 10). This indicates the importance of the flow unsteadiness upon the swirl distortion characteristics. The S-duct unsteady flow is dominated by the Switching and Vertical POD Modes, which represent the swirl switching (Fig. $16 c, d)$ and the vertical oscillation associated with the unsteady centreline shear layer (Fig. 16a,b). Therefore these perturbations are expected to play a major role in the dynamic swirl distortion characteristics. To quantify the effect of these flow features upon the swirl distortion metrics, the unsteady flow field is re-constructed using all the modes except for the pertinent mode considered. The swirl distortion descriptors are then evaluated from the modified flow field in the absence of that particular mode.

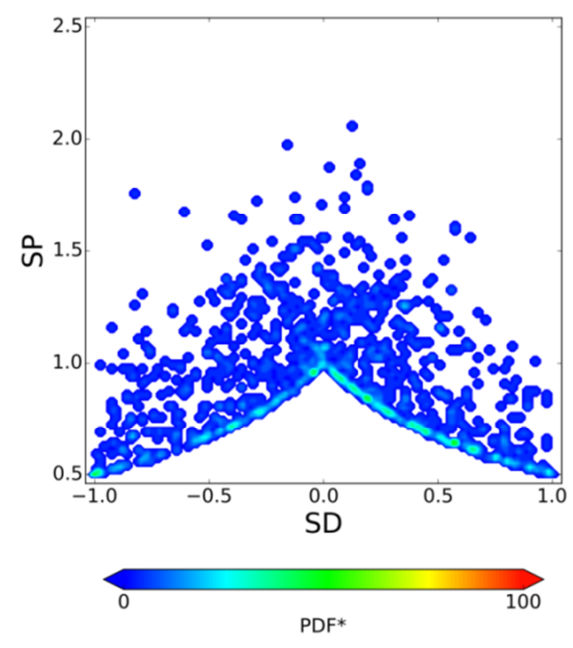

(a) Duct-1 $(\mathrm{h} / \mathrm{L}=0.50)$

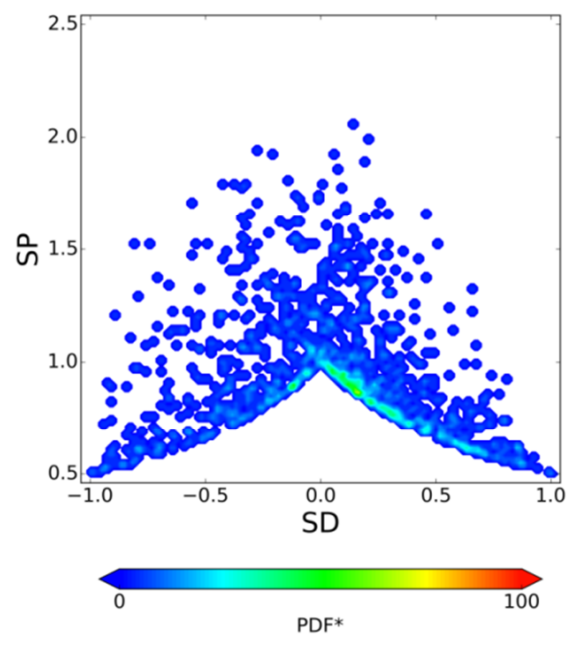

(b) Duct-2 $(\mathrm{h} / \mathrm{L}=0.27)$

Fig. 19 SP-SD joint-PDF when the Switching Mode is subtracted $\left(r / R=0.32, M_{r e f}=0.27\right)$

For Duct-1 $(\mathrm{h} / \mathrm{L}=0.50)$ the number of bulk swirl events is significantly reduced when the Switching Mode (SM) is subtracted (Fig. 19a) compared to the original flow field (Fig. 8a). To quantify the effect of the different coherent structures on the swirl pattern, the probability of bulk-swirl and twin-swirl events are obtained as the integral PDF 
value within the areas $\mathrm{SP}=[0.5,0.7] \times|\mathrm{SD}|=[0.8,1.0]$ and $\mathrm{SP}=[0.9,1.1] \times \mathrm{SD}=[0.8,1.0]$, respectively, and compared with the original-flow values (Table 2). The Switching Mode promotes most of the bulk swirl events associated with the $\mathrm{SI}_{\max }$ for both configurations. About $60 \%$ of the original bulk-swirl events in the high offset Duct-1 are removed when the $\mathrm{SM}$ is absent, and $\mathrm{SI}_{\max }$ reduces from $25^{\circ}$ to $19.6^{\circ}$ (Table 2). The number of twin-swirl events is almost insensitive to the presence of this mode. For Duct-2 $(\mathrm{h} / \mathrm{L}=0.27)$ the $\mathrm{SM}$ accounts for a lower proportion of bulk swirl events compared to the high offset Duct-1 (Fig. 19b). Only 36\% of the original bulk swirl events require the presence of the $\mathrm{SM}$ to occur, while the $\mathrm{SI}_{\max }$ is also significantly reduced from $17.1^{\circ}$ to $13.7^{\circ}$. For Duct-2 (h/L=0.27) the SM is also associated with the presence of twin-swirl patterns in the inner ring $(r / R=0.32)$. About $30 \%$ of the original twin-swirl events are not observed in the absence of this perturbation. The number of bulk swirl events and $\mathrm{SI}_{\max }$ are insensitive to the presence of the Vertical Mode (Table 2). In contrast, this perturbation accounts for most of the twin-swirl patterns for both configurations. About $50 \%$ and $65 \%$ of the original-flow twin-swirl events are only observed in the presence of this flow feature, for Duct- $1(\mathrm{~h} / \mathrm{L}=0.50)$ and Duct-2 $(\mathrm{h} / \mathrm{L}=0.27)$ respectively. When both SM and VM are removed from the flow field, high SI values still occur in the inner ring $(\mathrm{r} / \mathrm{R}=0.32)$ for both configurations (Table 2). Therefore the dynamic swirl distortion is also affected by higher order POD modes, which represent less-energetic flow features.

Table 2 POD modes impact on the swirl distortion characteristics at the inner-most $\operatorname{ring}(r / R=0.32)$

\begin{tabular}{|c|c|c|c|c|c|c|}
\hline \multirow[b]{2}{*}{ Mode removed } & \multicolumn{3}{|c|}{ Duct-1 $(\mathrm{h} / \mathrm{L}=0.50) \mathrm{M}_{\mathrm{ref}}=0.27$} & \multicolumn{3}{|c|}{ Duct-2 $(\mathrm{h} / \mathrm{L}=0.27) \mathrm{M}_{\text {ref }}=0.27$} \\
\hline & $\begin{array}{l}\mathbf{P}_{\text {bulk }} \\
(\%)\end{array}$ & $\begin{array}{l}\mathbf{P}_{\text {twin }} \\
(\%)\end{array}$ & $\begin{array}{c}\mathbf{S I}_{\max } \\
\left(^{\circ}\right)\end{array}$ & $\begin{array}{l}\mathbf{P}_{\text {bulk }} \\
(\%)\end{array}$ & $\begin{array}{l}\mathbf{P}_{\text {twin }} \\
(\%)\end{array}$ & $\begin{array}{c}\mathbf{S I}_{\max } \\
\left(^{\circ}\right)\end{array}$ \\
\hline None & 22.4 & 9.4 & 25.0 & 7.5 & 14.3 & 17.1 \\
\hline Switching & 9.5 & 8.8 & 19.6 & 4.8 & 9.8 & 13.7 \\
\hline Vertical & 24.0 & 4.6 & 25.5 & 7.6 & 4.8 & 17.0 \\
\hline Switching and vertical & 11.2 & 3.5 & 18.8 & 6.0 & 2.8 & 12.2 \\
\hline
\end{tabular}

\section{Conclusions}

The unsteady three-component velocity field for two S-duct configurations with a different centreline offset has been measured at the AIP using S-PIV. The highly unsteady nature of the flow was quantified, which indicates that the unsteady flow field is substantially different from the time-averaged flow. The flow unsteadiness promotes peak 
values of swirl distortion which are one order of magnitude greater compared to the static value evaluated from the mean flow. In addition, the swirl distortion pattern deviates from the typical twin-swirl distribution indicated by the mean flow, and even bulk-swirl patterns are observed. This highlights the limitation of time-averaged data obtained with low-bandwidth probes or RANS simulations.

POD is applied on the three components of the measured velocity vector field to identify the fundamental coherent structures of the AIP flow field. The most-energetic coherent structures for both ducts are the vertical and switching modes. The vertical mode is postulated to be associated with the unsteadiness of the centreline shear layer. The switching mode represents a perturbation of the circumferential velocity field, and is responsible for the swirl switching mechanism by which one of the Dean vortices becomes dominant and leads to high levels of swirl intensity. The switching mode is associated with a circumferential modulation of the main loss region, which follows the movement of the dominant vortex. For the high offset duct the switching mode perturbation is sufficiently energetic to completely bias the in-plane flow field in one direction and promote single rotating cells, while these structures are rarely observed in the low offset duct. The impact of these coherent structures on the swirl distortion metrics has been quantified. The vertical mode is mainly associated with the twin-swirl patterns, and accounts for about half of the twin-swirl events for both configurations. The switching mode is more related to bulk swirl events which promote the greatest swirl intensity values.

The duct offset has a strong impact on the unsteady swirl distortion characteristics and the mean, standarddeviation and maximum values of swirl intensity are substantially greater in the high offset duct compared to the low offset configuration. The joint-PDF maps presented in this work show that the swirl distortion pattern in the low offset duct oscillates around the most-probable twin swirl distribution which is observed in the mean flow, and deviations towards bulk-swirl patterns are relatively rare. In contrast, the high offset duct shows similar frequency of occurrence for negative bulk swirl, twin swirl and positive bulk swirl events. For both configurations the most intense distortion events in terms of swirl intensity are promoted by bulk swirl patterns. Patterns with multiple swirling structures are also observed in both ducts, even though these events are less frequent and the associated swirl intensity values are relatively lower compared to those associated with bulk swirl patterns. The $\alpha_{1}$ and $\theta_{1}$ descriptors used in this work are defined as the greatest sector swirl in the swirl angle distribution and the associated circumferential extent, and highlight distortion patterns for which the swirling flow extends over a small area of the section. Peak values of $\alpha_{1}$ similar to the maximum swirl intensity are observed which are associated with extents 
between $60^{\circ}-200^{\circ}$ and $60^{\circ}-140^{\circ}$ for the high and low offset ducts, respectively. These high-swirl events are not highlighted by the usual swirl distortion descriptors SI, SP and SD. However they are important since the associated extents exceed the estimated critical angle of $60^{\circ}-90^{\circ}$, and could adversely affect the stability of the engine and should be considered for the intake-engine compatibility assessment. This work shows that the most intense swirl distortion events occur at the inner ring for both high and low offset ducts

Overall this work indicates the impact of the duct offset in the unsteady swirl distortion characteristics. The main coherent structures of the flow have been identified and the associated perturbations in the in-plane and out-of-plane velocity fields have been analysed. The impact of these structures upon the swirl distortion characteristics has been quantified. The swirl distortion patterns associated with the greatest swirl intensities have been identified for the high and low offset configurations. The $\alpha_{l}$ and $\theta_{1}$ descriptors have been used to identify potent, local distortion events which are otherwise not revealed by the conventional SI, SP and SD descriptors.

\section{References}

[1] Kawai, R. T., Friedman, D. M., and Serrano, L., "Blended Wing Body (BWB) Boundary Layer Ingestion (BLI) Inlet Configuration and System Studies,” NASA Technical Report CR-2006-214534, Dec. 2006.

[2] Kim, H., and Liou, M., "Flow Simulation of N2B Hybrid Wing Body Configuration," 50th AIAA Aerospace Sciences Meeting including the New Horizons Forum and Aerospace Exposition, AIAA 2012-0838, Nashville, Tennessee, USA, Jan. 2012. Doi: $10.2514 / 6.2012-838$

[3] Society of Automotive Engineers, "A Methodology for Assessing Inlet Swirl Distortion," SAE Aerospace Information Report 5686, Warrendale, PA, USA, 2007.

[4] Society of Automotive Engineers, "Inlet Total-Pressure-Distortion Considerations for Gas-Turbine Engines," SAE Aerospace Information Report 1419a, Warrendale, PA, USA, 1999.

[5] Kidman, D. S., Reagan, P. V., and Malloy, D. J., "Comparison of Inlet Compatibility Results From Subscale Wind Tunnel and Full-Scale Flight Tests of the F/A-22 Aircraft with the F119-PW-100 Engine," 17th Symposium on Air Breathing Engines, International Society of Air Breathing Engines, ISABE-2005-1169, 2005.

[6] Wenzel, L. M., and Blaha, R. J., “Analysis of Dynamic Inlet Distortion Applied to a Parallel Compressor Model,” NASA TM X-3522, May 1977.

[7] Rademakers, R. P. M., Bindl, S., and Niehuis, R., "Effects of Flow Distortions as They Occur in S-Duct Inlets on the Performance and Stability of a Jet Engine,” ASME Journal of Engineering for Gas Turbines and Power, Vol. 138, No. 2, Sep. 2015, p. 022605. Doi: 10.1115/1.4031305 
[8] Cousins, W., T., "History, Philosophy, Physics and Future Directions of Aircraft Propulsion System / Inlet Integration," Proceedings of ASME Turbo Expo 2004: Power for Land, Sea, and Air, Vol. 2, GT2004-54210, June 2004, pp. 305-320. Doi: $10.1115 / G T 2004-54210$

[9] Bowditch, D. N., and Coltrin, R. E., “A Survey of Inlet/Engine Distortion Compatibility,” NASA TM-83421, June 1983.

[10] Stevens, C. H., Spong, E. D., and Hammock, M. S., "F-15 Inlet/Engine Test Techniques and Distortion Methodologies Studies. Volume 1: Technical Discussion,” NASA CR-144866, June 1978.

[11] Wellborn, S. R., Reichert, B. A., and Okiishi, T. H., "Study of the Compressible Flow in a Diffusing S-Duct," AIAA Journal of Propulsion and Power, Vol. 10, No. 5, Sept.-Oct. 1994, pp. 668-675. Doi: 10.2514/3.23778

[12] Fiola, C., and Agarwal, K. R., "Simulation of Secondary and Separated Flow in Diffusing S Ducts," AIAA Journal of Propulsion and Power, Vol. 31, No. 1, Jan-Feb 2015. Doi: 10.2514/1.B35275

[13] Berens, T. M., Delot, A. L., Chevalier, M., and Van Muijden, J., "Numerical Simulations for High Offset Intake Diffuser Flows," 52nd AIAA Aerospace Sciences Meeting - AIAA SciTech 2014, AIAA 2014-0371, National Harbor, Maryland, USA, Jan. 2014 . Doi: 10.2514/6.2014-0371

[14] Garnier, E., "Flow Control by Pulsed Jet in a Curved S-Duct: A Spectral Analysis," AIAA Journal, Vol. 53, No. 10, Oct. 2015, pp. 2813-2827. Doi: 10.2514/1.J053422

[15] Zachos, P. K., MacManus, D. G., Gil-Prieto, D., and Chiereghin, N., "Flow Distortion Measurements in Convoluted Aeroengine Intakes,” AIAA Journal, Vol. 54, No. 9, Sep. 2016, pp. 2819-2832. Doi: 10.2514/1.J054904

[16] MacManus, D. G., Chiereghin, N., Gil Prieto, D., Zachos, P., "Complex Aero-Engine Intake Ducts and Dynamic Distortion," $33^{\text {rd }}$ AIAA Applied Aerodynamics Conference, AIAA Aviation, AIAA 2015-3304, Dallas, TX, 2015. Doi: $10.2514 / 6.2015-3304$

[17] Kalpakli Vester, A., Örlü, R., and Alfredsson, P. H., "POD analysis of the turbulent flow downstream a mild and sharp bend," Experiments in Fluids, Vol. 56, No. 3, Article id. 57, 15 pp., March 2015. Doi: 10.1007/s00348-015-1926-6

[18] Tunstall, M. J., and Harvey, J. K., "On the effect of a sharp bend in a fully developed turbulent pipe-flow," Journal of Fluid Mechanics, Vol. 34, No. 3, Dec. 1968, pp. 595-608. Doi: 10.1017/S0022112068002107

[19] Rütten, F., Schröder, W., and Meinke, M., "Large-eddy simulation of low frequency oscillations of the Dean vortices in turbulent pipe bend flows," Physics of Fluids, Vol. 17, No. 3, 035107, 2005. Doi: 10.1063/1.1852573

[20] Hellström, L. H. O., Zlatinov, M. B., Cao, G., and Smits, A. J., “Turbulent Pipe Flow Downstream of a 90 Bend,” Journal of Fluid Mechanics, Vol. 735, R7, Oct. 2013, pp. 1-12. Doi: 10.1017/jfm.2013.534

[21] Samuelsson, I., "Test of an UCAV air inlet duct (Eikon) at static conditions in FOI suckdown facility," Swedish Defence Research Agency (FOI), Technical report FOI-R-1572--SE, Nov. 2005. ISSN 1650-1942. 
[22] Hodder, B. K., “An Investigation of Engine Influence on Inlet Performance”, NASA CR-166136, 1981.

[23] Motycka, D. L., "Reynolds Number and Fan/Inlet Coupling Effects on Subsonic Transport Inlet Distortion”, J. Propulsion and Power, vol. 1, pp. 229-234, 1985. Doi: 10.2514/3.22785

[24] Raffel, M., Willert, C., Wereley, S., and Kompenhans, J., Particle Image Velocimetry: A Practical Guide, $2^{\text {nd }}$ ed., SpringerVerlag, Berlin, 2007, Chap. 5, pp. 164-176. Doi: 10.1007/978-3-540-72308-0

[25] Kostas, J., Soria, J., and Chong, M. S., “A comparison between snapshot POD analysis of PIV velocity and vorticity data,” Experiments in Fluids, Vol. 38, No. 2, 2005, pp. 146-160. doi: 10.1007/s00348-004-0873-4

[26] Kostas, J., Soria, J., Chong, and M. S., "Particle image velocimetry measurements of a backward-facing step flow," Experiments in Fluids, Vol. 33, No. 6, 2002, pp. 838-853. Doi: 10.1007/s00348-002-0521-9

[27] Chen, H., Reuss, D. L., Hung, D. L., and Sick, V., “A practical guide for using proper orthogonal decomposition in engine research,” International Journal of Engine Research, Vol. 14, No. 4, 2013, pp. 307-319. Doi: 10.1177/1468087412455748

[28] Gurka, R., Liberzon, A., and Hetsroni, G., "POD of vorticity fields: A method for spatial characterization of coherent structures," International Journal of Heat and Fluid Flow, Vol. 27, No. 3, June 2006, pp. 416-423. Doi: 10.1016/j.ijheatfluidflow.2006.01.001

[29] Holmes, P., Lumley, J. L., Berkooz, G., and Rowley, C. W., Turbulence, Coherent Structures, Dynamical systems and Symmetry, $2^{\text {nd }}$ ed., Cambridge University Press, Cambridge, England, UK, 2012, Chap. 3.

[30] Lumley, J. L., Stochastic Tools in turbulence, Dover ed., Dover Publications, Inc., New York, USA, 2007, Chap. 3, pp. 5759.

[31] Berkooz, G., Holmes, P., and Lumley, J. L., "The proper orthogonal decomposition in the analysis of turbulent flows," Annual Review of Fluid Mechanics, Vol. 25, 1993, pp. 539-575. Doi: 10.1146/annurev.fl.25.010193.002543

[32] Sirovich, L., "Turbulence and the dynamics of coherent structures. Part 1: Coherent structures," Quaterly of Applied Mathematics, vol. XLV, Oct. 1987, pp. 561-571.

[33] Mitchell, G. A., "Effect of Inlet Ingestion of a Wing Tip Vortex on Compressor Face Flow and Turbojet Stall Margin," Tech. Rep. TM X-3246, NASA, 1975.

[34] Doane, D. P., and Seward, L. E., "Measuring Skewness : A Forgotten Statistic?," Journal of Statistics Education, Vol. 19, No. 2, 2011, pp. 1-18.

[35] Balanda, K. P., and MacGillivray, H. L., "Kurtosis: A Critical Review," The American Statistician, Vol. 42, No. 2, May 1988, pp. 111-119. Doi: 10.1080/00031305.1988.10475539

[36] Pope, S. B., Turbulent Flows, Cambridge University Press, Cambridge, England, UK, 2000, Chap. 3, pp. $40,56$. 
[37] Gordeyev, S. V., and Thomas, F. O., "Coherent structure in the turbulent planar jet. Part 2. Structural topology via POD eigenmode projection,” Journal of Fluid Mechanics, Vol. 460, 2002, pp. 349-380. Doi: 10.1017/S0022112002008364

[38] Davis, M., Beale, D., and Sheoran, Y., "Integrated Test and Evaluation Techniques As Applied To an Inlet Swirl Investigation Using the F109 Gas Turbine Engine," Proceedings of ASME Turbo Expo 2008, Power for Land, Sea, and Air, Vol. 1, GT2008-50074, June 2008, pp. 9-20. Doi: 10.1115/GT2008-50074 
2017-04-18

\section{Convoluted intake distortion}

\section{measurements using stereo particle image velocimetry}

Gil-Prieto, Daniel

Daniel Gil-Prieto, David G. MacManus, Pavlos K. Zachos, Geoffrey Tanguy and Kevin R.

Menzies. Convoluted intake distortion measurements using stereo particle image velocimetry. AIAA Journal, Vol. 55, No. 6 (2017), pp1878-1892

http://dx.doi.org/10.2514/1.J055467

Downloaded from Cranfield Library Services E-Repository 